\title{
Robust Control for Autonomous Spacecraft Evacuation with Model Uncertainty and Upper Bound of Performance with Constraints
}

\author{
Dian Sheng, ${ }^{1}$ Xuebo Yang, ${ }^{2}$ and Hamid Reza Karimi ${ }^{3}$ \\ ${ }^{1}$ Space Control and Inertial Technology Research Center, Harbin Institute of Technology, Harbin 150001, China \\ ${ }^{2}$ Bohai University, Jinzhou 121013, China \\ ${ }^{3}$ Department of Engineering, Faculty of Engineering and Science, University of Agder, 4898 Grimstad, Norway
}

Correspondence should be addressed to Xuebo Yang; yangxuebo@gmail.com

Received 4 September 2013; Accepted 30 October 2013; Published 8 April 2014

Academic Editor: Zhiguang Feng

Copyright (C) 2014 Dian Sheng et al. This is an open access article distributed under the Creative Commons Attribution License, which permits unrestricted use, distribution, and reproduction in any medium, provided the original work is properly cited.

\begin{abstract}
This paper studies the problem of guaranteed cost control for spacecraft evacuation. The relative dynamic model is established based on Clohessy-Wiltshire (C-W) equations. The paper has taken parameter uncertainty, output tracking, disturbance attenuation, and fuel cost into consideration. The paper introduces a new Lyapunov approach, so the controller design problem can be transferred into a convex optimization problem subject to linear matrix inequality (LMI) constraints. By using the controller, the spacecraft evacuation can be completed in a safe extent. Meanwhile, the fuel cost also has an upper bound. Then the paper analyzes the approach of evacuation and discusses possible initial states of the spacecraft for the controller design. An illustrative example is applied to show the effectiveness of the proposed control design method, and different performances caused by different initial states of spacecraft (-V-bar, -R-bar, and $+\mathrm{H}$-bar) are simulated.
\end{abstract}

\section{Introduction}

With the development of aerospace science, the research of space exploration is deepening gradually. Among them, manned space technology has been in the limelight of the aerospace around the world. In addition, most tracking spacecrafts need to be evacuated safely after completing autonomous spacecrafts rendezvouz. Furthermore, evacuation segment can be divided into three stages: unlocking, separation, and orbital transformation. In the study of evacuation, Fehse has introduced the process and the bounds of the evacuation [1]. Besides, he has also compared the evacuation from V-bar and R-bar and has discussed the security of the two means. Yin et al. also review most popular data-driven PM-FD methods with recent developments [2, 3]. Hablani et al. has studied the target spacecraft by using a kind of multiplepulse linear guidance control method applicable to arbitrary direction approach and evacuation [4]. Bergez et al. has reached the conclusion which is based on an assumption, where the safe evacuation theory is happening in the failure of capture lock while the ATV is docking with the Russian Mir space station [5]. However, some correlative issues have not yet been fully explained because of their complexity and uncertainty, and many existing studies have left considerable room for improvement. This leads us to look for a new method to control the evacuation phase of spacecraft.

Moreover, spacecraft relative motion problems are often took over based on Clohssey and Wiltshire (C-W) equations in $1960[6,7]$. Generally speaking, the equations are transformed into a state function based on $\dot{\mathbf{x}}(t)=A \mathbf{x}(t)+$ $B \mathbf{u}(t)$, where $\mathbf{x}(t)$ is the relative position and velocity states vector and $\mathbf{u}(t)$ is the control input vector. This description has been used widely to study the spacecraft rendezvous problems [8-11]. But due to many uncertain factors, the state matrix $A$ and the control input matrix $B$ are not easy to be determined accurately. Besides, the elements of matrix $A$ are related to the angle velocity of the target spacecraft which is susceptible to many inevitable factors such as errors of detection. Besides, the conceivable mass variation of fuel causes the input of thrusters inaccuracy, which can be 
regarded as the uncertainty of the input matrix $B$ [12]. These uncertainties may lessen stability of spacecraft evacuation phase. In the past decades, many researches about uncertain system papers [13-18]. Petersen presents an algorithm for the stabilization of a class of uncertain linear systems. The uncertain systems under consideration are described by state equations which depend on time-varying unknown-butbounded uncertain parameters [19]. Singla et al. has developed an output feedback structured model reference adaptive control law for spacecraft rendezvous and docking problems [20]. However, the parameter uncertainties have not attracted enough attention to the research of spacecraft evacuation phase control problems. This leads to our desire to think over uncertainties and find a proper method to handle them.

The evacuation phase and short-range phase all belong to the relative navigation phase, both of them based on the relative phase. Evacuation phase is an opposite movement process to short-range phase with the final approach phase. Spacecraft rendezvous and docking are unusual complex fields of aerospace work, which must be measured accurately to the relative position and relative attitude of the two spacecrafts. Rendezvous and docking phases ask for a very high requirement of accuracy, reliability, and control system robustness of the independent measurement system. The slightest mistake could result in docking failure. However, the researches of the spacecraft evacuation seem too few compared with the researches of spacecraft rendezvous and docking. That is because spacecraft evacuation needs less automaticity than rendezvous, which is primarily based on orbit control to ensure the security of the process. In that case, the chaser will not collide with the target spacecraft even when the thrust equipment is out of control. So the study of spacecraft rendezvous and docking has a significant reference for the control of evacuation phase.

Besides, rendezvous and docking not only need the orbital maneuvering control, but also are based on the advanced attitude control. Nevertheless, the evacuation phase is mainly based on the orbital maneuvering control. To sum up, research of orbital maneuvering control for the rendezvous has a very important significance of the evacuation phase. In recent 50 years, related researchers have done indepth research for spacecraft rendezvous and docking and made a lot of research achievements in the orbital maneuvering control problem. In addition, spacecraft autonomous rendezvous (RVD) has been recognized as a crucial issue for many progressive astronautic missions. Besides, spacecraft autonomous rendezvous is also very important in the field of the manned space flight project. Manned space flight project generally requires the technique of spacecraft autonomous rendezvous, such as the spacecraft orbit service, space rescue, repair, and the space station supplies. Nowadays, United States, Russia, Europe, Japan, and other countries are involving to the area of spacecraft autonomous rendezvous and have experiment successfully for hundreds of times. As manned space technology develops, the theory of spacecraft autonomous rendezvous will be more mature than the past [8-11, 20, 21].

The paper provides a systematic research of the control problem aiming at the orbit of the spacecraft autonomous

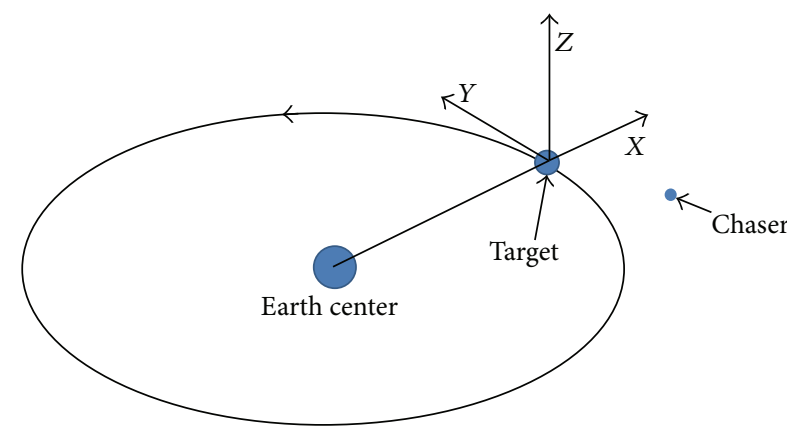

FIGURE 1: Spacecraft rendezvous and the utilized coordinate.

evacuation and gives a new method to control the evacuation phase of spacecraft. Based on the two-body problem, the paper has constructed the relative motion equation for the process of evacuation, which is the C-W equation, and makes a brief introduction about the bound that the spacecraft has to comply with. Then it gives the new controller calculated by the bound of guaranteed cost output [22-27]. Firstly, the paper analyzes the characteristic of the holding point. Then it uses the state error variables to replace the state vector. Transform the tracking problem of constant reference signal into the problem of asymptotically stability under the feedback control [28]. However, the spacecraft has to receive many disturbances during the flight, such as solar radiation pressure perturbation and electromagnetic radiation. Thus the paper takes the uncertainty into consideration when it designs the controller. Besides, the paper considers the limited constraint condition and the quadratic performance index, transforms the problem into convex optimization of the LMI by the method of Lyapunov, and then receives the guaranteed cost output with the minimal upper bound. Verify the solution by simulation with MATLAB. In the end of the paper, we compare the fuel consumers under the different levels of the disturbance and simulate the output tracking of the chaser spacecraft by three cases (-V-bar, -Rbar, and $+\mathrm{H}$-bar). The result proves that the model has good dynamic response, reliability, and self-adaptability. Therefore, the model can be applied to control system of spacecraft.

\section{Problem Formulation}

A right-handed Cartesian coordinate can be established based on the structure of the target. As shown in Figure 1, the origin attaches to the mass center of the target, the $x$-axis is along the vector from earth center to the origin, the $y$-axis is along the target orbit circumference, and the $z$-axis sets up the right-handed frame [29].

In this coordinate system, the evacuation phase described in this paper can be depicted in Figure 2.

Define $R_{0}$ as the radius of the target circular orbit and $n$ as the angular velocity of the target equals $\left(\mu_{e} / R_{0}^{3}\right)^{1 / 2}$, where $\mu_{e}$ is the gravitational parameter of the earth. Considering the $\mathrm{C}-\mathrm{W}$ equations and proposing the mathematical description 


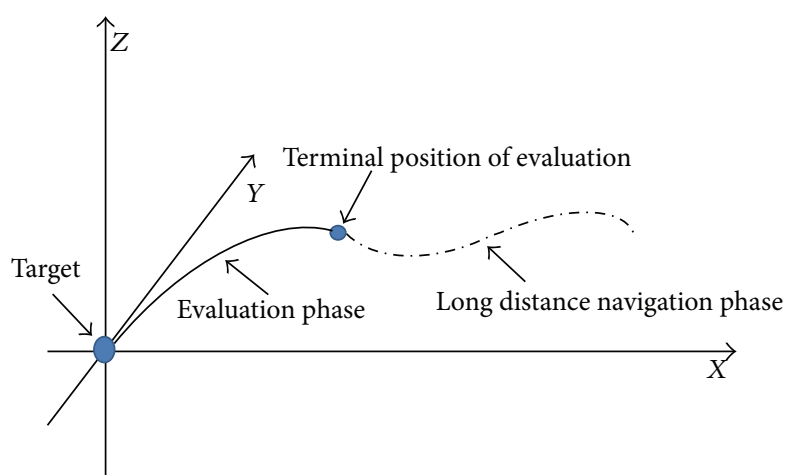

Figure 2: Evacuation phases of rendezvous in the target frame coordinate.

of the relative motion for two adjacent spacecrafts, the relative dynamic model can be described by C-W's equations as

$$
\begin{gathered}
\ddot{x}-2 n \dot{y}-3 n^{2} x=\frac{1}{m} T_{x}, \\
\ddot{y}+2 n \dot{x}=\frac{1}{m} T_{y}, \\
\ddot{z}+n^{2} z=\frac{1}{m} T_{z},
\end{gathered}
$$

where $x, y$, and $z$ are the components of the relative position in corresponding axes, $m$ is the mass of the chaser, and $T_{i}(i=$ $x, y, z)$ is the $i$ th component of the control thrust applied on the spacecraft [30].

2.1. Transfer of Evacuation Phase. By defining the state vector $x(t)=[x, y, z, \dot{x}, \dot{y}, \dot{z}]^{T}$, control input vector $u(t)=$ $\left[T_{x}, T_{y}, T_{z}\right]^{T}$, and output vector $y(t)=[x, y, z]^{T}$, we have

$$
\begin{gathered}
\dot{x}(t)=A x(t)+B u(t), \\
y(t)=C x(t),
\end{gathered}
$$

where

$$
\begin{gathered}
A=\left[\begin{array}{cccccc}
0 & 0 & 0 & 1 & 0 & 0 \\
0 & 0 & 0 & 0 & 1 & 0 \\
0 & 0 & 0 & 0 & 0 & 1 \\
3 n^{2} & 0 & 0 & 0 & 2 n & 0 \\
0 & 0 & 0 & -2 n & 0 & 0 \\
0 & 0 & -n^{2} & 0 & 0 & 0
\end{array}\right], \\
B=\left[\begin{array}{ccc}
0 & 0 & 0 \\
0 & 0 & 0 \\
0 & 0 & 0 \\
\frac{1}{m} & 0 & 0 \\
0 & \frac{1}{m} & 0 \\
0 & 0 & \frac{1}{m}
\end{array}\right], \\
C=\left[\begin{array}{lll}
1 & 0 & 0 \\
0 & 1 & 0 \\
0 & 0 & 1 \\
0 & 0 & 0 \\
0 & 0 & 0 \\
0 & 0 & 0
\end{array}\right] .
\end{gathered}
$$

Thus, the specific relative motion of chaser and target can be realized by designing proper control input thrust $u(t)$.

2.2. Uncertainty. Due to the measure the complex external perturbations among the objects in space, the target angle velocity $n$ can be described accurately. Besides, inevitable input uncertainties still exist because of the error of the thrust and fuel. Having taken these modeling uncertainties into consideration, the system functions can be expressed as follows:

$$
\begin{gathered}
\dot{x}(t)=(A+\Delta A) x(t)+(B+\Delta B) u(t), \\
y(t)=C x(t) .
\end{gathered}
$$

The two norm-bounded uncertain matrices $\Delta A$ and $\Delta B$ have proper dimensions and are in the form of $\left[\begin{array}{ll}\Delta A & \Delta B\end{array}\right]=$ $\lambda \varphi(t)\left[\begin{array}{ll}\sigma_{1} & \sigma_{2}\end{array}\right]$, where $\lambda, \sigma_{1}$, and $\sigma_{2}$ are the constant matrices with proper dimensions, which can reflect the uncertainty structure; $\varphi(t)$ is an unknown real-time varying matrix with Lebesgue measurable elements bounded by $\varphi^{T}(t) \varphi(t) \leq I$. The introduced matrices can be regarded as the following patterns because of the structures of $A$ and $B$ :

$$
\begin{gathered}
\lambda=\Upsilon \times\left[\begin{array}{llllll}
0 & 0 & 0 & 1 & 0 & 0 \\
0 & 0 & 0 & 0 & 1 & 0 \\
0 & 0 & 0 & 0 & 0 & 1
\end{array}\right], \\
\sigma_{1}=\Xi \times\left[\begin{array}{llllll}
0 & 0 & 0 & 1 & 1 & 0 \\
1 & 0 & 0 & 1 & 0 & 0 \\
0 & 0 & 0 & 0 & 0 & 0
\end{array}\right], \\
\sigma_{2}=\Omega \times\left[\begin{array}{llll}
0 & 0 & 0 \\
0 & 0 & 0 \\
1 & 1 & 1
\end{array}\right]
\end{gathered}
$$

Define $\varphi(t)=\operatorname{diag}\left[\varphi_{1}(t), \varphi_{2}(t), \varphi_{3}(t)\right]$, where $\varphi_{1}(t), \varphi_{2}(t)$, and $\varphi_{3}(t)$ are three varying scalars within the boundary of $[0,1]$. And $\Upsilon, \Xi$, and $\Omega$ signify magnitudes of the uncertainties.

Then, the system state functions with modeling uncertainty can be rewritten as

$$
\begin{gathered}
\dot{x}(t)=\widetilde{A} x(t)+\widetilde{B} u(t), \\
y(t)=C x(t),
\end{gathered}
$$

where $\widetilde{A}=A+\lambda \varphi(t) \sigma_{1}$ and $\widetilde{B}=B+\lambda \varphi(t) \sigma_{2}$. It can be seen that matrices $\lambda, \sigma_{1}$, and $\sigma_{2}$ reflect the structural information of uncertainties.

2.3. Output Tracking. Considering the evacuation phase of spacecraft rendezvous, the terminal position of the chaser is a certain point that can be defined as a fixed reference output signal $x_{r}=\left(x_{r}, y_{r}, z_{r}, 0,0,0\right)^{T}$. Therefore, the evacuation phase orbital control problem can be solved by designing an output tracking controller. The output $x(t)$ of the closed-loop system tracks the reference signal $x_{r}$, which means that

$$
\lim _{t \rightarrow \infty}\left[x(t)-x_{r}\right]=0 .
$$


We introduce the error integral action in the controller for eliminating the steady-state tracking error. Then we define

$$
e(t)=\int_{0}^{t}\left[x(\tau)-x_{r}\right] d \tau
$$

and then we have $\dot{e}(t)=x(t)-x_{r}$.

Therefore, we obtain the augmented system

$$
\begin{gathered}
\dot{\xi}(t)=\widetilde{\Lambda} \xi(t)+\widetilde{\Gamma} u(t)+\widetilde{\Psi} \xi_{r}, \\
f(t)=\widetilde{\Phi} \xi(t)+\widetilde{\Phi} \xi_{r},
\end{gathered}
$$

where

$$
\begin{gathered}
\widetilde{\Lambda}=\left[\begin{array}{ll}
\widetilde{A} & 0 \\
C & 0
\end{array}\right], \quad \widetilde{\Gamma}=\left[\begin{array}{c}
\widetilde{B} \\
0
\end{array}\right], \quad \widetilde{\Psi}=\left[\begin{array}{c}
0 \\
-I
\end{array}\right], \\
\widetilde{\Phi}=\left[\begin{array}{ll}
C & 0
\end{array}\right], \quad \xi(t)=\left[\begin{array}{l}
q(t) \\
e(t)
\end{array}\right] .
\end{gathered}
$$

Considering the uncertainties described in (4), the augmented matrices $\widetilde{\Lambda}$ and $\widetilde{\Gamma}$ can be further transformed into $\widetilde{\Lambda}=\Lambda+\Delta \Lambda, \widetilde{\Gamma}=\Gamma+\Delta \Gamma$, where

$$
\begin{gathered}
\Lambda=\left[\begin{array}{ll}
A & 0 \\
C & 0
\end{array}\right], \quad \Gamma=\left[\begin{array}{c}
B \\
0
\end{array}\right], \quad \Delta \Lambda=\Pi \varphi(t) \sigma_{1 A}, \\
\Delta \Gamma=\Pi \varphi(t) \sigma_{2}, \quad \Pi=\left[\begin{array}{c}
D \\
0
\end{array}\right], \quad \sigma_{1 A}=\left[\begin{array}{ll}
E_{1} & 0
\end{array}\right] .
\end{gathered}
$$

Use the state feedback control law, and define $K=$ $\left[\begin{array}{ll}K_{q} & K_{d}\end{array}\right]$, then we obtain $u(t)=K \xi(t)=K_{q} q(t)+K_{d} d(t)$.

Then the augmented closed-loop system can be described as

$$
\dot{\xi}(t)=[\widetilde{\Lambda}+\widetilde{\Gamma} K] \xi(t)+\widetilde{\Psi} f_{r}
$$

The output tracking requirement in (7) can be satisfied if the closed-loop system in (12) is stable. Thus, the output tracking control problem studied in this paper can be transformed into the stabilization problem of the system in (9). If there has a controller $K$ guarantee the system stable in (9), then the output $\varphi(t)$ of (6) can track the reference signal $f_{r}$.

2.4. Control Performance. Primarily, we take the rendezvous control performances into consideration. Because of the weight boundary of spacecraft, the minimal fuel cost has to be chosen as one control performance index for evacuation. Then, by defining a control weighting matrix $R_{3 \times 3}$, the fuel cost performance index can be expressed as $J_{1}=$ $\int_{0}^{\infty} u^{T}(t) R u(t) d t$.

Secondly, there should not be violent shake during the orbital transfer for the security. Define a control weighting matrix $Q$, and then the smooth transfer trajectory control performance index can be written as $J_{2}=\int_{0}^{\infty} \xi^{T}(t) Q \xi(t) d t$.

Then, consider the two performance indexes together. The comprehensive control performance cost can be described as

$$
J=J_{1}+J_{2}=\int_{0}^{\infty}\left[u^{T}(t) R u(t)+\xi^{T}(t) Q \xi(t)\right] d t .
$$

For $u(t)=K \zeta(t)$, the equality (13) can be redescribed as

$$
J=\int_{0}^{\infty}\left[\zeta^{T}(t) K^{T} R K \zeta(t)+\zeta^{T}(t) Q \zeta(t)\right] d t .
$$

Then, the guaranteed cost control design problem can be depicted as follows: to find an admissible controller $K$, which makes the system performance cost $J$ meets a minimal upper bound $\mu$.

Besides, the dynamic performance of a system is correlated to the location of its poles, and the satisfactory transient responses can be ensured by constraining the poles to position in a prescribed region. In this paper, we consider the disk regional poles constraint, which has been proved efficient in both theory and practice. Consider the uncertain rendezvous dynamic model (9); design a guaranteed cost output tracking controller $K$, such that the closed-loop system in (12) is asymptotically stable (meaning that the output tracking requirement in (7) is satisfied); meanwhile, the performance cost in (14) is below a prescribed upper bound for all admissible uncertainties.

\subsection{Description of the Control Law}

Lemma 1. To the given symmetric matrix $A=\left[\begin{array}{ll}A_{11} & A_{12} \\ A_{21} & A_{22}\end{array}\right]$, the following conditions are equivalent:

(i) $A<0$;

(ii) $A_{11}<0, A_{22}-A_{12}^{T} A_{11}^{-1} A_{12}<0$;

(iii) $A_{22}<0, A_{11}-A_{12} A_{11}^{-1} A_{12}^{T}<0$.

Lemma 2. $u(t)=K x(t)$ is a guaranteed cost controller if there exist symmetric positive-definite matrices $P, S \in R^{3 \times 3}$ such that for all uncertain matrices $\varphi$ satisfying $\varphi^{T}(t) \varphi(t) \leqslant I$,

$$
\left[\begin{array}{cc}
\Sigma & P\left[\widetilde{A}+\lambda \varphi\left(\sigma_{1}+\sigma_{2}\right)\right] \\
{\left[\widetilde{A}+\lambda \varphi\left(\sigma_{1}+\sigma_{2}\right)\right]^{T} P} & -S
\end{array}\right]<0,
$$

where $\Sigma=Q+K^{T} R K+S+\operatorname{sym}\left\{\mathrm{P}\left[\mathrm{A}+\mathrm{BK}+\lambda \varphi\left(\sigma_{1}+\sigma_{2}\right)\right]\right\}$ [31].

Based on Lyapunov stable theory, the controller design requirements such as input constraint and the guaranteed cost are formulated as some LMI conditions, and the controller design problem is cast into a convex optimization problem subject to the LMI constraints.

Presume $x_{r}=0$. Define the Lyapunov function $V(\xi(t))=$ $\xi(t)^{T} P \xi(t)$, where $P$ is a positive symmetry matrix. Then,

$$
\dot{V}(\xi(t))=\xi(t)^{T} \operatorname{sym}\{P(\widetilde{\Lambda}+\widetilde{\Gamma} K)\} \xi(t) .
$$

If a controller $K$ can satisfy the following equation,

$$
\operatorname{sym}\{P(\widetilde{\Lambda}+\widetilde{\Gamma} K)\}<0,
$$

then the system begins to stabilize.

Besides, the target function should satisfy some constraints like Lemma 2 as follows:

$$
\dot{V}(\xi(t))<-\xi^{T}(t)\left(Q+K^{T} R K\right) \xi(t)<0 .
$$


Then the system not only begins to stabilize, but also has a certain upper constraint.

Consider the following:

$$
\begin{aligned}
J & =\int_{0}^{\infty}\left[\xi^{T}(t) Q \xi(t)+u^{T}(t) R u(t)\right] d t \\
& <v(\xi(0))=\xi^{T}(0) P \xi(0),
\end{aligned}
$$

where $\xi(0)$ means the error in the system.

The quadratic performance index is restrained by $V(\xi(e))=\xi(t)^{T} P \xi(t)$. This upper bound form of the performance index can qualitatively describe the consumption of fuel and concussion of trace.

The function can be transferred as based on Lemma 2 .

Consider the following:

$$
\operatorname{sym}\{P(\widetilde{\Lambda}+\widetilde{\Gamma} K)\}+Q+K^{T} R K<0 .
$$

However $P$ and $K$ are not independent. Therefore the function above is not a LMI. But based on Lemma 1 and define $\chi=\operatorname{sym}\{P(\widetilde{\Lambda}+\widetilde{\Gamma} K)\}, \varrho=\operatorname{diag}\left(R^{-1}, Q^{-1}\right)$, and $\omega=\left[K^{T}, I\right]$, it equals

$$
\left[\begin{array}{ll}
\chi & \omega \\
* & \varrho
\end{array}\right]<0
$$

Define $X=P^{-1}, Y=K P^{-1}, ð=\operatorname{sym}\{\widetilde{\Lambda} X+\widetilde{\Gamma} Y\}$, and $\widehat{H}=\left[Y^{T}, I\right]$. We obtain the function

$$
\left[\begin{array}{cc}
\text { ð } & \widehat{H} \\
* & \varrho
\end{array}\right]<0 .
$$

The function is a LMI about $X$ and $Y$ to the certain system matrix $\widetilde{\Lambda}$ and the input matrix $\widetilde{\Gamma}$. Using the LMI box in the Matlab can readily solve the function.

First, introduce the matrix $U_{x}=[1,0,0]^{T}[1,0,0] ; U_{y}=$ $[0,1,0]^{T}[0,1,0] ; U_{z}=[0,0,1]^{T}[0,0,1]$.

Then, we obtain

$$
\begin{aligned}
& u_{x}^{2}(t)=\left[U_{x} u(t)\right]^{T}\left[U_{x} u(t)\right] \leqslant u_{x, \max }^{2} \\
& u_{y}^{2}(t)=\left[U_{y} u(t)\right]^{T}\left[U_{y} u(t)\right] \leqslant u_{y, \max }^{2} \\
& u_{z}^{2}(t)=\left[U_{z} u(t)\right]^{T}\left[U_{z} u(t)\right] \leqslant u_{z, \max }^{2}
\end{aligned}
$$

where $u_{x, \max }, u_{y, \text { max }}$, and $u_{z \text {, max }}$ are the maximum inputs of the system.

According to $u(t)=K \xi(t)$, we have the constraint of the system

$$
\begin{aligned}
u_{i}^{2}(t) & =\left[U_{i} K \xi(t)\right]^{T}\left[U_{i} K \xi(t)\right] \\
& =\xi^{T}(t) K^{T} U_{i}^{T} U_{i} K \xi(t) \leqslant u_{i, \max }^{2},
\end{aligned}
$$

where $i=x, y, z$.
Because the LMI has already guaranteed that the closed system has the upper bound, then

$$
V(\xi(t))=\xi(t)^{T} P \xi(t)<V(\xi(0)) .
$$

Thus, when the $x_{r}$ has been given, the function $V(\xi(0))=$ $\xi(0)^{T} P \xi(0)$ has the constraint $V(\xi(0))<\mu$.

Then, we have

$$
u_{i, \max }^{-2} \xi^{T}(t) K^{T} U_{i}^{T} U_{i} K \xi(t)<\mu^{-1} \xi^{T}(t) P \xi(t) .
$$

Because we know if the target constraint is less than the Lyapunov function, the controller can satisfy the requirement. Then, we obtain

$$
u_{i, \max }^{-2} K^{T} U_{i}^{T} U_{i} K<\mu^{-1} P .
$$

However, the function above is not the LMI. According to Lemma 1, we have

$$
\left[\begin{array}{cc}
-\mu^{-1} P & \left(U_{i} K\right)^{T} \\
* & -u_{i, \max }^{2}
\end{array}\right]<0 .
$$

Take the left and right by matrix $\operatorname{diag}\left\{P^{-1}, I\right\}$, then the LMI can be described as follows:

$$
\left[\begin{array}{cc}
-\mu^{-1} X & \left(U_{i} Y\right)^{T} \\
* & -u_{i, \max }^{2}
\end{array}\right]<0 .
$$

Besides, we can alter the function above:

$$
\left[\begin{array}{cc}
-\mu & \xi(0)^{T} \\
* & -X
\end{array}\right]<0 .
$$

By solving the LMI above and using the $(X, Y)$, we can obtain the matrix $K$, then we can design the controller $u(t)$, in which $K=Y X^{-1}$.

Meanwhile, we can also obtain the upper bound of the system

$$
J_{\max }=\xi(0) X^{-1} \xi(0) .
$$

Next, we consider the obtained performance cost upper bound. Apart from the obtainable upper bounds of the performance consumers, it is also hopeful to make the bounds as low as possible to the practical engineering. We introduce another matrix $\Theta$ satisfying

$$
\left[\begin{array}{cc}
-\Theta & I \\
* & -X
\end{array}\right]<0
$$

which means $\Theta>X^{-1}>0$. Then, the lower performance cost bound can be obtained by solving the following optimization problem:

$$
\min \xi(0)^{T} \Theta \xi(0)
$$

s.t. LMIs (22), (29), (30) and (32). 
According to the results shown above, we can find that the solution of the optimization problem does not totally depend on the exact value of the uncertain matrix $\varphi(t)$, and the magnitudes of the uncertainties can be adjusted by changing the values of $\Upsilon, \Xi$, and $\Omega$. Thus, the effects of the parameter errors can be analyzed according to these three parameters. Particularly, when we assume $\Upsilon=\Xi=\Omega=0$, which means that there is no parameter error in the model, the uncertain matrices $\lambda, \sigma_{1}$, and $\sigma_{2}$ will be transformed into zero matrices. At the same time, the system takes exactly the form of the nominal system of no uncertainty. Correspondingly, the LMI constraints will alter, and the relative optimization problem can also be solved readily. The conditions are all formulated in the form of linear matrix inequalities, and the controller design is transformed into a convex optimization problem subject to LMI constraints that can be solved by Matlab. However, the conditions we obtained here are sufficient conditions for the existence of a proper controller. Thus, even if there is no parameter error, the result is not the unique solution of the controller design problem. This is because the guaranteed cost and the poles constraint of the closedloop system are considered simultaneously. It is still hard to solve this kind of multiobjective optimization problem, and finding a necessary and sufficient condition for the existence of the proper controller is difficult. However, the correlative problems are significant and worth investigating in our further studies. For the orbital control system, there are many other performances that can be adopted for the controller design. The orbital controller design problems with these kinds of performance measures for spacecraft rendezvous are worth studying in the future.

\section{Illustrative Example}

Because the target spacecraft has 3 methods to evacuation (-V-bar, -R-bar, and +H-bar), this chapter discussed and simulated the guaranteed cost control low based on these methods separately. In this section, we provide an example to illustrate the usefulness and advantage of the controller design method proposed in the above sections. We consider a couple of adjacent spacecrafts, where the chaser is being transferred towards the target along the homing phase orbit. Assume the mass of the chaser is $600 \mathrm{~kg}$, and the target is moving in a geosynchronous orbit of radius $r=42241 \mathrm{~km}$ with an orbital period of 24 hours. Thus, we have the angle velocity $n=1.117 \times 10^{-3} \mathrm{rad} / \mathrm{s}$. Assume that the maximum input control force is $130 \mathrm{~N}$.

3.1. -V-bar. According to the coordinate based on target frame, we presume that the chaser transfers to position $(135,0,0)$. Therefore, the initial error state can be expressed as $(-135,0,0,0,0,0,0,0,0)^{T}$.

For simplicity, we presume the thrust can vary continuously. First, we analyze the situation with $\Upsilon=\Xi=\Omega=$ 0.03 . In the coordinate based on target frame, assume the initial state of homing phase is $[0,0,0,0,0,0,0,0,0]$. And by considering the construction of uncertainties in the form of (9), we make the following assumptions:

$$
\begin{gathered}
X=\left[\begin{array}{cccccccccc}
0.0001 & 0.0000 & 0.0000 & -0.0000 & -0.0000 & -0.0000 & -0.0020 & -0.0001 & -0.0001 \\
0.0000 & 0.0001 & 0.0000 & -0.0000 & -0.0000 & -0.0000 & -0.0003 & -0.0020 & -0.0005 \\
0.0000 & 0.0000 & 0.0001 & -0.0000 & -0.0000 & -0.0000 & -0.0002 & -0.0005 & -0.0021 \\
-0.0000 & -0.0000 & -0.0000 & 0.0000 & 0.0000 & 0.0000 & 0.0000 & 0.0000 & 0.0000 \\
-0.0000 & -0.0000 & -0.0000 & 0.0000 & 0.0000 & 0.0000 & 0.0000 & 0.0000 & 0.0000 \\
-0.0000 & -0.0000 & -0.0000 & 0.0000 & 0.0000 & 0.0000 & 0.0000 & 0.0000 & 0.0000 \\
-0.0020 & -0.0003 & -0.0002 & 0.0000 & 0.0000 & 0.0000 & 0.0626 & 0.0029 & 0.0015 \\
-0.0001 & -0.0020 & -0.0005 & 0.0000 & 0.0000 & 0.0000 & 0.0029 & 0.0604 & 0.0076 \\
-0.0001 & -0.0005 & -0.0021 & 0.0000 & 0.0000 & 0.0000 & 0.0015 & 0.0076 & 0.0621
\end{array}\right], \\
Y=10^{-3} \times\left[\begin{array}{cccccccccc}
-0.0198 & -0.0002 & -0.0001 & -0.0002 & 0.0000 & 0.0000 & 0.3065 & -0.0200 & -0.0097 \\
0.0053 & -0.0063 & -0.0058 & -0.0002 & -0.0005 & -0.0003 & -0.1446 & 0.0986 & 0.0035 \\
0.0075 & -0.0050 & -0.0061 & -0.0002 & -0.0003 & -0.0006 & -0.1492 & -0.0014 & 0.0904
\end{array}\right] .
\end{gathered}
$$

Therefore, the gain matrix for the augmented feedback controller is given by

$$
K=Y \times X^{-1}=\left[\begin{array}{ccccccccc}
-0.8725 & 0.0156 & -0.0023 & -19.6879 & -0.0475 & -0.0355 & -0.0156 & -0.0000 & -0.0002 \\
-0.1172 & -0.7895 & -0.2242 & -1.8656 & -21.4237 & -1.1423 & -0.0032 & -0.0140 & -0.0073 \\
0.0174 & -0.1945 & -0.7776 & -0.5884 & -0.2998 & -20.7360 & -0.0010 & -0.0066 & -0.0142
\end{array}\right]
$$




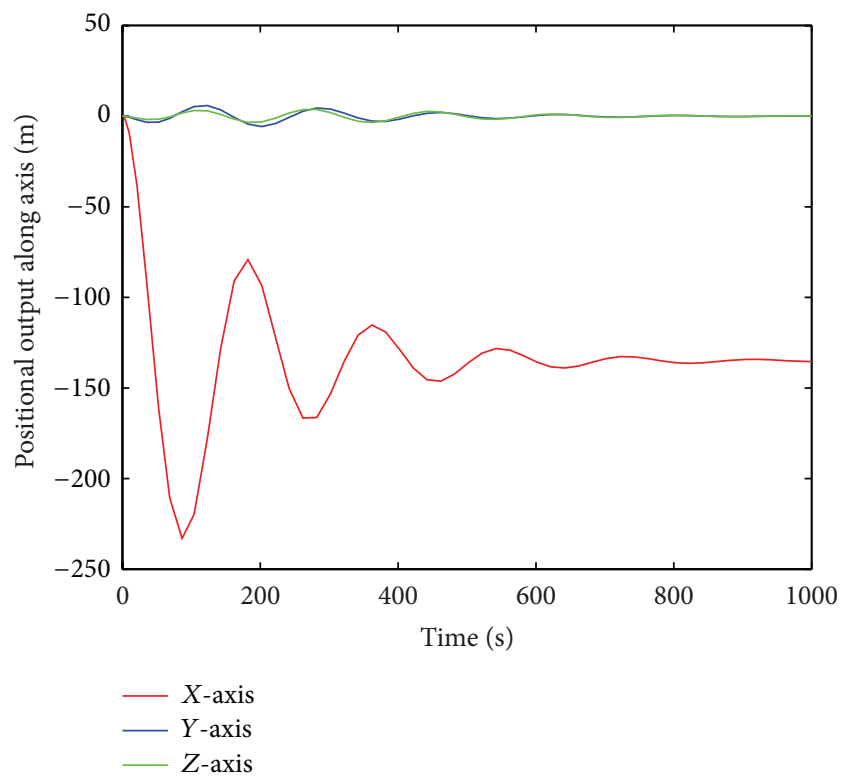

FIGURE 3: Chaser's positional output of closed-loop control.

The output of the system which means the relative position of the two spacecrafts is depicted in Figure 3.

It can be seen that the output of the system is asymptotically convergent to the reference signal, which means that the chaser keeps at the terminal point. We can also obtain the relevant control thrusts needed for these methods in Figure 4.

Besides the nonzero initial velocity, the coupling action brought by parameter uncertainties is another source for the fluctuation in $z$-axis. It can be seen that the fluctuation finally restrained and the positional output in $z$-axis asymptotically converged to the reference signal. We can see that the controller can effectively stabilize the system in spite of the existence of parameter uncertainties.

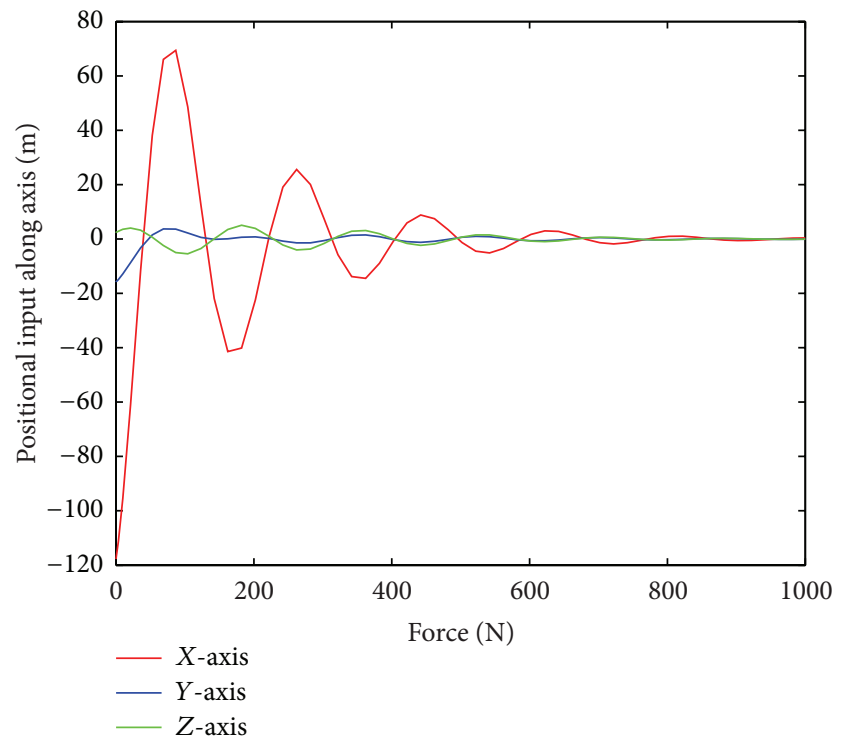

FIGURE 4: Chaser's input thrust of closed-loop control.

Next, we change the magnitudes of uncertainties by considering the following four conditions:

$$
\begin{aligned}
& \text { Case A: } \Upsilon=\Xi=\Omega=0 ; \\
& \text { Case B: } \Upsilon=0.03, \quad \Xi=\Omega=0.015 \text {; } \\
& \text { Case C: } \Upsilon=0.06, \quad \Xi=\Omega=0.03 ; \\
& \text { Case D: } \Upsilon=\Xi=\Omega=0.0652 .
\end{aligned}
$$

The proper controllers for these conditions can be obtained by solving the convex optimization problems as follows:

$$
\begin{aligned}
K_{\text {Case A }} & =\left[\begin{array}{ccccccccc}
-0.8726 & 0.0168 & -0.0012 & -19.6631 & -0.0165 & -0.0238 & -0.0156 & 0.0001 & -0.0001 \\
-0.0510 & -0.8183 & -0.1570 & -0.7472 & -22.4896 & -0.5307 & -0.0021 & -0.0140 & -0.0052 \\
-0.0232 & -0.1522 & -0.8247 & -1.0570 & -0.5274 & -22.3842 & -0.0012 & -0.0052 & -0.0144
\end{array}\right], \\
K_{\text {Case B }} & =\left[\begin{array}{ccccccccc}
-0.8728 & 0.0137 & -0.0035 & -19.6836 & -0.0418 & -0.0430 & -0.0157 & -0.0001 & -0.0003 \\
-0.0886 & -0.7852 & -0.2359 & -1.5512 & -21.3489 & -1.2784 & -0.0029 & -0.0140 & -0.0074 \\
-0.0103 & -0.2165 & -0.7832 & -1.1239 & -0.8532 & -20.8999 & -0.0015 & -0.0071 & -0.0143
\end{array}\right], \\
K_{\text {Case C }} & =\left[\begin{array}{ccccccccc}
-0.8723 & 0.0153 & -0.0014 & -19.7047 & -0.0793 & -0.0305 & -0.0156 & -0.0001 & -0.0002 \\
-0.1809 & -0.7652 & -0.2506 & -2.9144 & -20.5827 & -1.3472 & -0.0042 & -0.0138 & -0.0083 \\
0.0635 & -0.2401 & -0.7132 & -0.0714 & -0.5607 & -19.1985 & -0.0007 & -0.0075 & -0.0132
\end{array}\right], \\
K_{\text {Case D }} & =\left[\begin{array}{ccccccccc}
-0.8702 & 0.0296 & 0.0013 & -19.6788 & -0.0089 & 0.0016 & -0.0154 & 0.0004 & 0.0000 \\
-0.3263 & -0.7475 & -0.1084 & -2.9313 & -22.6185 & -0.2819 & -0.0036 & -0.0106 & -0.0041 \\
0.2335 & 0.0512 & -0.7417 & 3.9454 & 4.0154 & -19.6471 & 0.0032 & -0.0009 & -0.0129
\end{array}\right],
\end{aligned}
$$

and the positional outputs of the closed-loop systems with these controllers in three axes are depicted in Figures
5, 7, and 9 separately. We can also obtain the four relevant control thrusts needed for these two methods in 


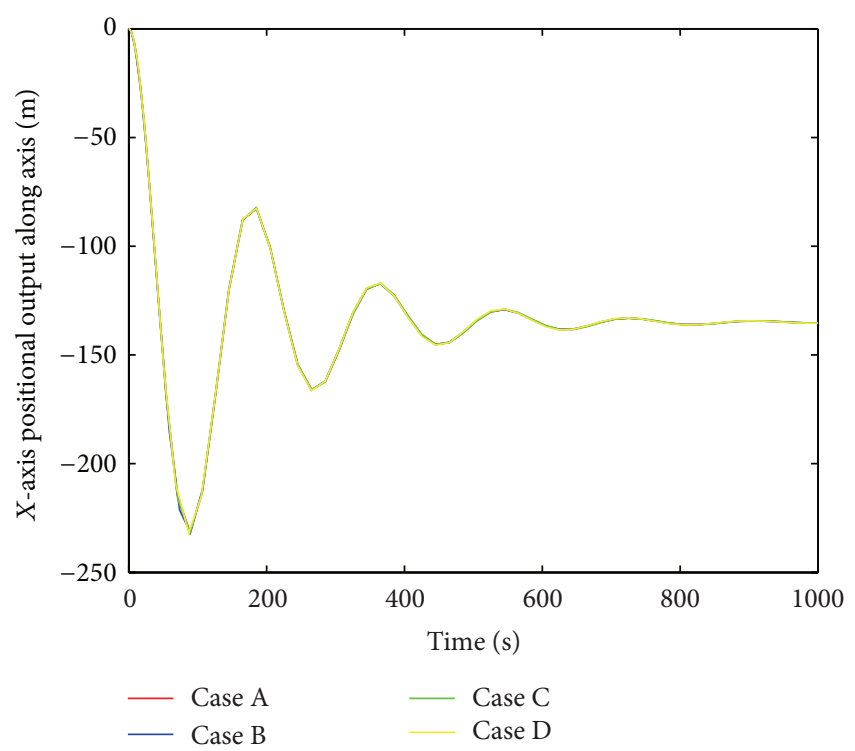

FIGURE 5: $X$-axis positional output of closed-loop system for the four different uncertainty cases.

Figures 6,8 , and 10 separately. We can see that the closedloop systems with the controllers $K$ are all stable and the output tracking requirement can be guaranteed. From these figures and the data, we can also find that the larger uncertainties will extend the stabilizing time and bring larger fluctuations in $z$-axis input thrust, and the performance cost bound will also be elevated by larger uncertainties.

Finally, the terminal segment close to the target is depicted clearly in Figure 11. It can be seen that the chaser will asymptotically move to the evacuation point eventually,

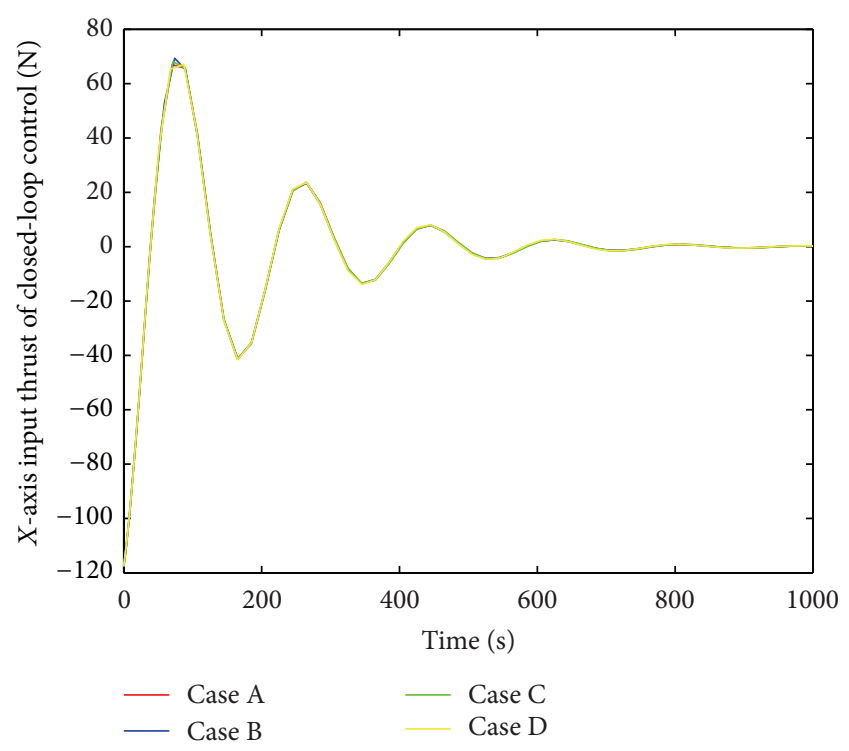

FIGURE 6: $X$-axis input of closed-loop system for the four different uncertainty cases.

which means that the output tracking requirements can be satisfied by the designed controller $K$.

3.2. -R-bar. According to the coordinate based on target frame, we presume that the chaser transfers to position $(0,-135,0)$. Therefore, the initial error state can be expressed as $(0,135,0,0,0,0,0,0,0)^{T}$.

For simplicity, we presume the thrust can vary continuously. First, we analyze the situation with $\Upsilon=\Xi=\Omega=$ 0.03 . In the coordinate based on target frame, assume the initial state of homing phase is $[0,0,0,0,0,0,0,0,0]$. And by considering the construction of uncertainties in the form of (9), we make the following assumptions:

$$
\begin{gathered}
X=\left[\begin{array}{cccccccccc}
0.0001 & 0.0000 & 0.0000 & -0.0000 & -0.0000 & -0.0000 & -0.0019 & -0.0002 & -0.0005 \\
0.0000 & 0.0001 & 0.0000 & -0.0000 & -0.0000 & -0.0000 & -0.0003 & -0.0020 & -0.0002 \\
0.0000 & 0.0000 & 0.0001 & -0.0000 & -0.0000 & -0.0000 & -0.0005 & -0.0002 & -0.0021 \\
-0.0000 & -0.0000 & -0.0000 & 0.0000 & 0.0000 & 0.0000 & 0.0000 & 0.0000 & 0.0000 \\
-0.0000 & -0.0000 & -0.0000 & 0.0000 & 0.0000 & 0.0000 & 0.0000 & 0.0000 & 0.0000 \\
-0.0000 & -0.0000 & -0.0000 & 0.0000 & 0.0000 & 0.0000 & 0.0000 & 0.0000 & 0.0000 \\
-0.0019 & -0.0003 & -0.0005 & 0.0000 & 0.0000 & 0.0000 & 0.0590 & 0.0035 & 0.0086 \\
-0.0002 & -0.0020 & -0.0002 & 0.0000 & 0.0000 & 0.0000 & 0.0035 & 0.0630 & 0.0028 \\
-0.0005 & -0.0002 & -0.0021 & 0.0000 & 0.0000 & 0.0000 & 0.0086 & 0.0028 & 0.0618
\end{array}\right], \\
Y=10^{-3} \times\left[\begin{array}{ccccccccc}
-0.0068 & 0.0065 & -0.0062 & -0.0005 & -0.0002 & -0.0003 & 0.0595 & -0.1188 & -0.0113 \\
-0.0004 & -0.0194 & -0.0005 & -0.0000 & -0.0002 & -0.0000 & -0.0014 & 0.2965 & -0.0121 \\
-0.0051 & 0.0077 & -0.0059 & -0.0003 & -0.0002 & -0.0006 & 0.0089 & -0.1633 & 0.1018
\end{array}\right] .
\end{gathered}
$$

Therefore, the gain matrix for the augmented feedback controller is given by

$$
K=Y \times X^{-1}=\left[\begin{array}{ccccccccc}
-0.8013 & -0.0073 & -0.2275 & -20.7499 & -2.1876 & -1.2217 & -0.0148 & -0.0009 & -0.0077 \\
-0.0521 & -0.8723 & -0.0134 & -0.1245 & -19.6957 & -0.0528 & -0.0009 & -0.0157 & -0.0007 \\
-0.2448 & 0.0160 & -0.7531 & -0.8687 & -0.3932 & -20.6737 & -0.0075 & -0.0008 & -0.0134
\end{array}\right]
$$




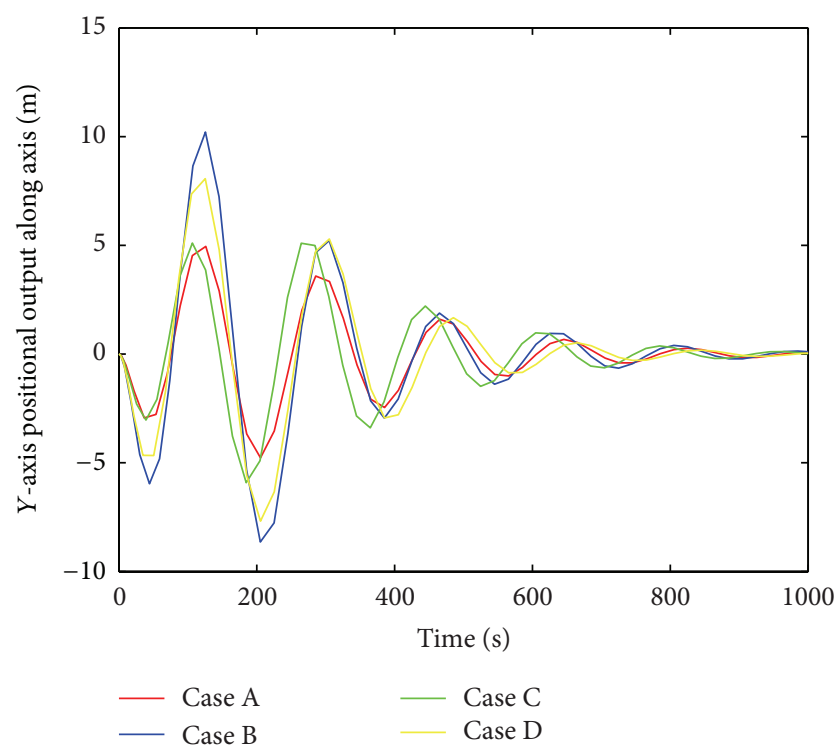

FIGURE 7: $Y$-axis positional output of closed-loop system for the four different uncertainty cases.

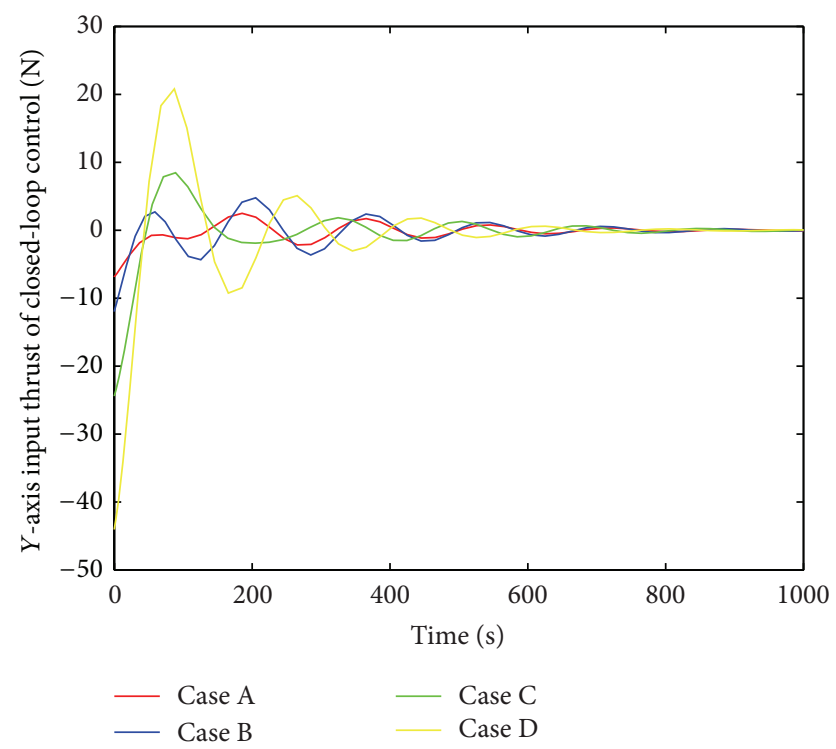

FIGURE 8: $Y$-axis input of closed-loop system for the four different uncertainty cases.

The output of the system which means the relative position of the two spacecraft is depicted in Figure 12.

It can be seen that the output of the system is asymptotically convergent to the reference signal, which means that the chaser keeps at the terminal point. We can also obtain the relevant control thrusts needed for these methods in Figure 13.

Besides the nonzero initial velocity, the coupling action brought by parameter uncertainties is another source for the fluctuation in $z$-axis. It can be seen that the fluctuation is finally restrained and the positional output in $z$-axis asymptotically converged to the reference signal. We can see that the controller can effectively stabilize the system in spite of the existence of parameter uncertainties.

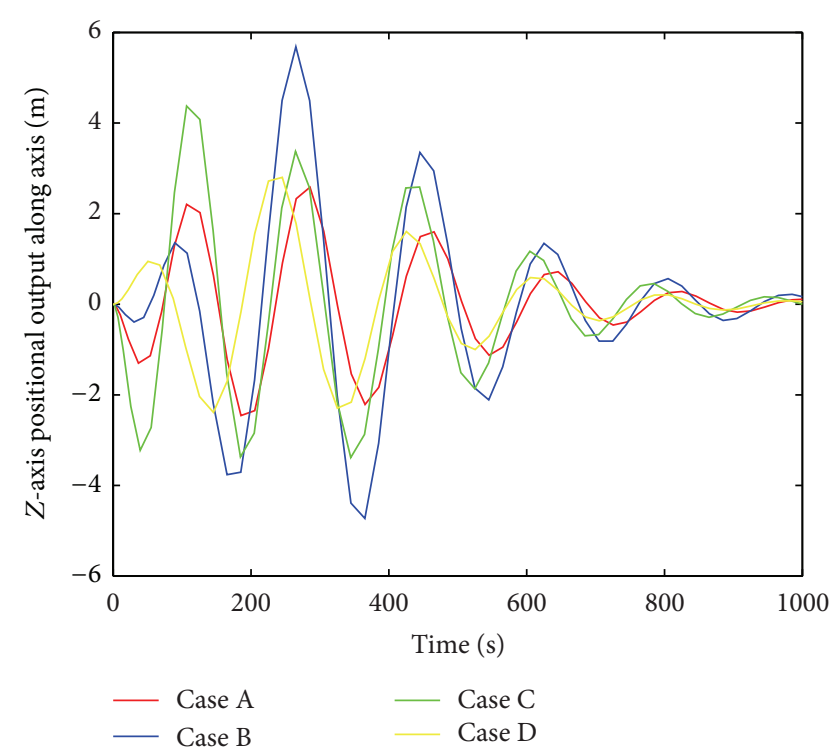

FIGURE 9: $Z$-axis positional output of closed-loop system for the four different uncertainty cases.

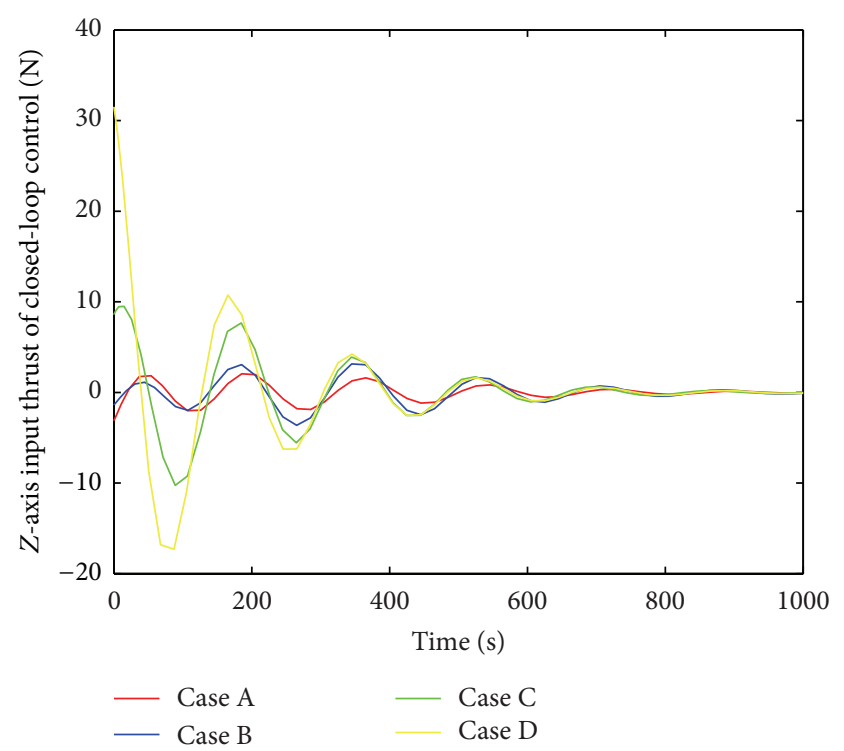

FIGURE 10: $Z$-axis input of closed-loop system for the four different uncertainty cases.

Next, we change the magnitudes of uncertainties by considering the following four conditions:

Case A: $\Upsilon=\Xi=\Omega=0$;

Case B: $\Upsilon=0.03, \quad \Xi=\Omega=0.015$;

Case C: $\Upsilon=0.06, \quad \Xi=\Omega=0.03$;

Case D: $\Upsilon=\Xi=\Omega=0.0652$.

The proper controllers for these conditions can be obtained by solving the convex optimization problems as follows: 


$$
\begin{aligned}
K_{\text {Case A }}= & {\left[\begin{array}{lllllllll}
-0.8255 & 0.0128 & -0.1707 & -22.1674 & -1.1565 & -0.6244 & -0.0146 & -0.0002 & -0.0058 \\
-0.0275 & -0.8714 & -0.0092 & -0.0469 & -19.6785 & -0.0279 & -0.0007 & -0.0156 & -0.0005 \\
-0.1763 & -0.0132 & -0.8192 & -0.6430 & -0.7610 & -22.3625 & -0.0058 & -0.0010 & -0.0143
\end{array}\right], } \\
K_{\text {Case B }}= & {\left[\begin{array}{lllllllll}
-0.7953 & -0.0035 & -0.2394 & -20.8135 & -1.9250 & -1.3488 & -0.0147 & -0.0009 & -0.0078 \\
-0.0425 & -0.8720 & -0.0144 & -0.1018 & -19.6821 & -0.0528 & -0.0010 & -0.0157 & -0.0007 \\
-0.2562 & -0.0016 & -0.7652 & -1.2303 & -0.7713 & -20.9066 & -0.0078 & -0.0011 & -0.0137
\end{array}\right], } \\
K_{\text {Case C }}= & {\left[\begin{array}{llllllllll}
-0.8103 & -0.0211 & -0.2089 & -20.2798 & -2.8811 & -1.0569 & -0.0151 & -0.0013 & -0.0076 \\
-0.0766 & -0.8730 & -0.0122 & -0.2170 & -19.7221 & -0.0484 & -0.0011 & -0.0158 & -0.0007 \\
-0.2670 & 0.0462 & -0.6986 & -1.1317 & 0.1392 & -19.6532 & -0.0075 & -0.0003 & -0.0122
\end{array}\right], } \\
K_{\text {Case D }}= & {\left[\begin{array}{lllllllll}
-0.8509 & -0.0492 & -0.1465 & -20.3975 & -4.1579 & -0.5316 & -0.0154 & -0.0016 & -0.0062 \\
-0.1433 & -0.8744 & -0.0109 & -0.5140 & -19.7942 & -0.0420 & -0.0014 & -0.0159 & -0.0007 \\
-0.1512 & 0.1218 & -0.6620 & 0.6308 & 2.0097 & -18.6886 & -0.0048 & -0.0013 & -0.0108
\end{array}\right], }
\end{aligned}
$$

and the positional outputs of the closed-loop systems with these controllers in three axes are depicted in Figures 14, 16 , and 18 separately. We can also obtain the four relevant control thrusts needed for these two methods in Figures 15, 17 , and 19 separately. We can see that the closed-loop systems with the controllers $K$ are all stable and the output tracking requirement can be guaranteed. From these figures and the table, we can also find that larger uncertainties will extend the stabilizing time and bring larger fluctuations in $z$-axis input thrust, and the performance cost bound will also be elevated by larger uncertainties.

Finally, the terminal segment close to the target is depicted clearly in Figure 20. It can be seen that the chaser will asymptotically move to the evacuation point eventually, which means that the output tracking requirements can be satisfied by the designed controller $K$.
3.3. + H-bar. Due to the poor security and the accident of collision, the application of the H-bar is less than those of the methods discussed above. However, $\mathrm{H}$-bar evacuation is easy to accomplish because the $z$-axis is uncorrelated with $x$-axis and $y$-axis. Besides, $\mathrm{H}$-bar evacuation consumers less fuel and can move along the $\mathrm{H}$-bar automatically. So, we consider $\mathrm{H}$-bar evacuation as a kind of illustrative example.

According to the coordinate based on target frame, we presume that the chaser transfers to position $(0,0,135)$. Therefore, the initial error state can be expressed as $(0,0,-135,0,0,0,0,0,0)^{T}$.

For simplicity, we presume the thrust can vary continuously. First, we analyze the situation with $\Upsilon=\Xi=\Omega=$ 0.03 . In the coordinate based on target frame, assume the initial state of homing phase is $[0,0,0,0,0,0,0,0,0]$. And by considering the construction of uncertainties in the form of (9), we make the following assumptions:

$$
\begin{gathered}
X=\left[\begin{array}{cccccccccc}
0.0001 & 0.0001 & 0.0000 & -0.0000 & -0.0000 & -0.0000 & -0.0017 & -0.0008 & -0.0004 \\
0.0001 & 0.0001 & 0.0000 & -0.0000 & -0.0000 & -0.0000 & -0.0010 & -0.0018 & -0.0004 \\
0.0000 & 0.0000 & 0.0001 & -0.0000 & -0.0000 & -0.0000 & -0.0003 & -0.0003 & -0.0021 \\
-0.0000 & -0.0000 & -0.0000 & 0.0000 & 0.0000 & 0.0000 & 0.0000 & 0.0000 & 0.0000 \\
-0.0000 & -0.0000 & -0.0000 & 0.0000 & 0.0000 & 0.0000 & 0.0000 & 0.0000 & 0.0000 \\
-0.0000 & -0.0000 & -0.0000 & 0.0000 & 0.0000 & 0.0000 & 0.0000 & 0.0000 & 0.0000 \\
-0.0017 & -0.0010 & -0.0003 & 0.0000 & 0.0000 & 0.0000 & 0.0541 & 0.0169 & 0.0056 \\
-0.0008 & -0.0018 & -0.0003 & 0.0000 & 0.0000 & 0.0000 & 0.0169 & 0.0559 & 0.0057 \\
-0.0004 & -0.0004 & -0.0021 & 0.0000 & 0.0000 & 0.0000 & 0.0056 & 0.0057 & 0.0638
\end{array}\right], \\
Y=10^{-3} \times\left[\begin{array}{ccccccccc}
-0.0070 & -0.0068 & 0.0019 & -0.0005 & -0.0004 & -0.0004 & 0.0599 & 0.0242 & -0.0729 \\
-0.0070 & -0.0070 & 0.0017 & -0.0004 & -0.0004 & -0.0004 & 0.0306 & 0.0444 & -0.0716 \\
-0.0024 & -0.0023 & -0.0180 & -0.0001 & -0.0001 & -0.0003 & -0.0459 & -0.0436 & 0.2483
\end{array}\right] .
\end{gathered}
$$

Therefore, the gain matrix for the augmented feedback controller is given by

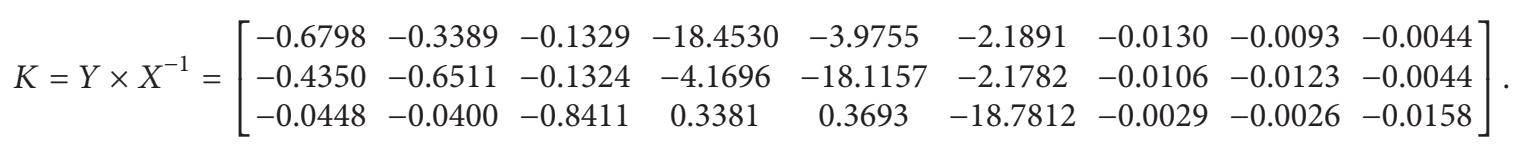




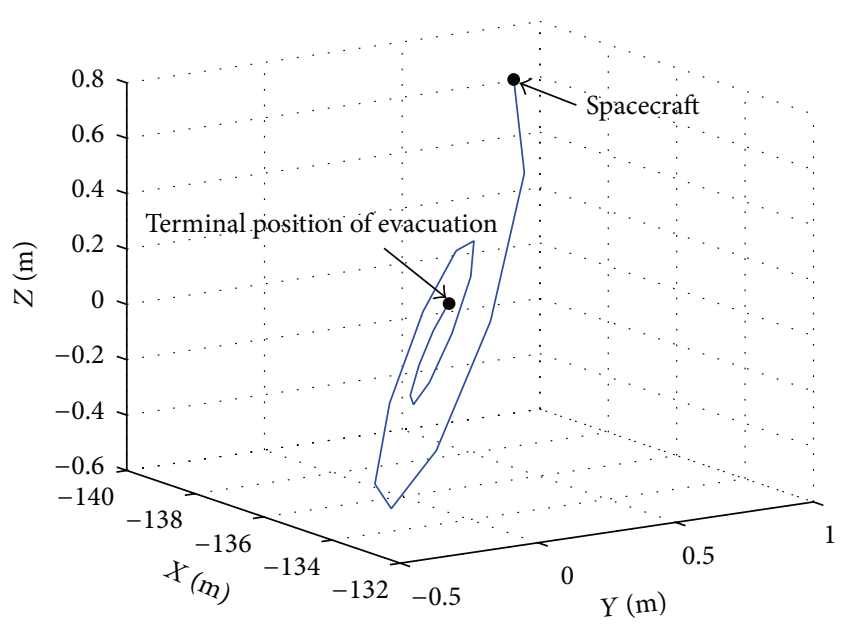

FIGURE 11: Terminal segment of the transfer orbit in rendezvous homing phase.

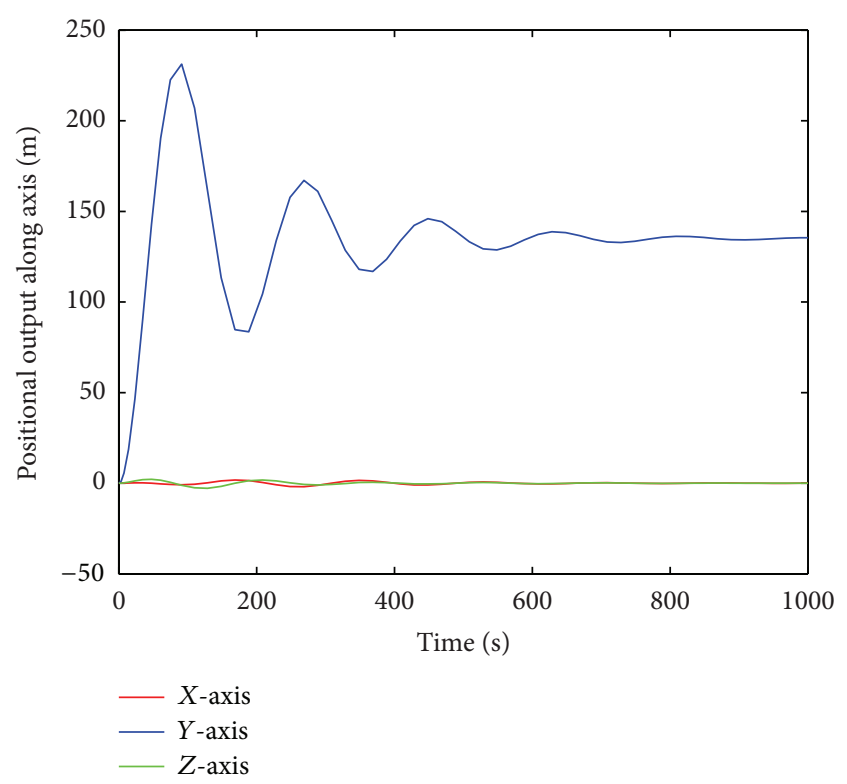

FIGURE 12: Chaser's positional output of closed-loop control.

The output of the system which means the relative position of the two spacecrafts is depicted in Figure 21.

It can be seen that the output of the system is asymptotically convergent to the reference signal, which means that the chaser keeps at the terminal point. We can also obtain the relevant control thrusts needed for these methods in Figure 22.

Besides the nonzero initial velocity, the coupling action brought by parameter uncertainties is another source for the fluctuation in $z$-axis. It can be seen that the fluctuation finally restrained and the positional output in $z$-axis asymptotically converged to the reference signal. We can see that the controller can effectively stabilize the system in spite of the existence of parameter uncertainties.

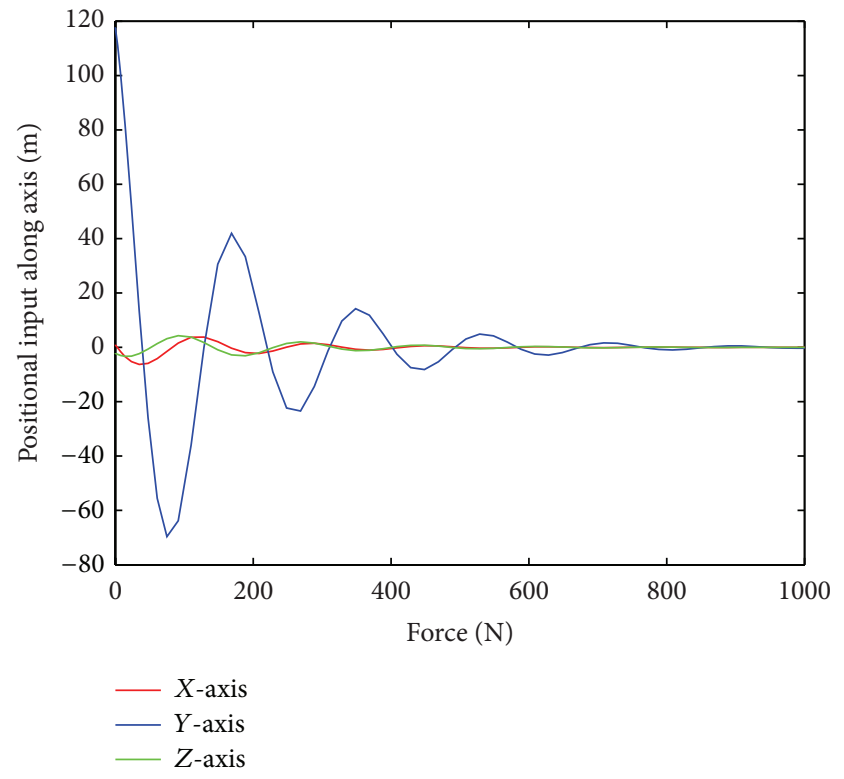

FIGURE 13: Chaser's input thrust of closed-loop control.

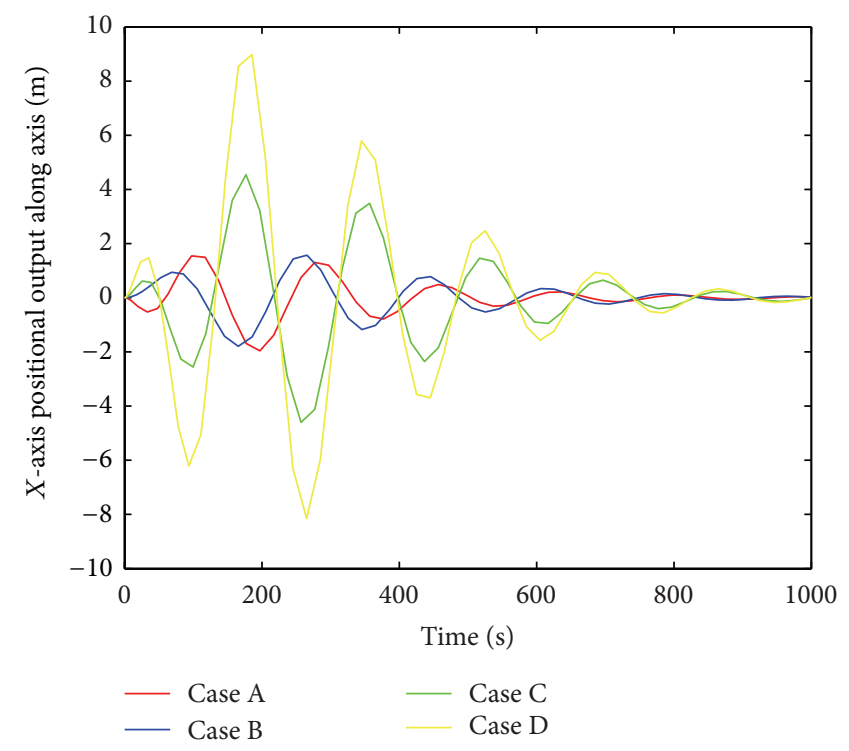

FIgURE 14: $X$-axis positional output of closed-loop system for the four different uncertainty cases.

Next, we change the magnitudes of uncertainties by considering the following four conditions:

$$
\begin{aligned}
& \text { Case A: } \Upsilon=\Xi=\Omega=0 ; \\
& \text { Case B: } \Upsilon=0.03, \quad \Xi=\Omega=0.015 ; \\
& \text { Case C: } \Upsilon=0.06, \quad \Xi=\Omega=0.03 ; \\
& \text { Case D: } \Upsilon=\Xi=\Omega=0.0652 .
\end{aligned}
$$

The proper controllers for these conditions can be obtained by solving the convex optimization problems as follows: 


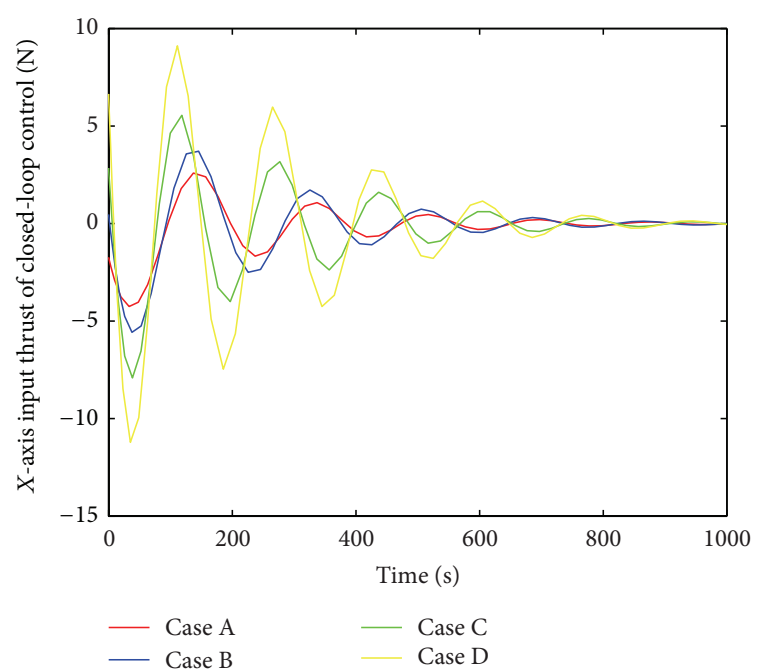

FIGURE 15: $X$-axis input of closed-loop system for the four different uncertainty cases.

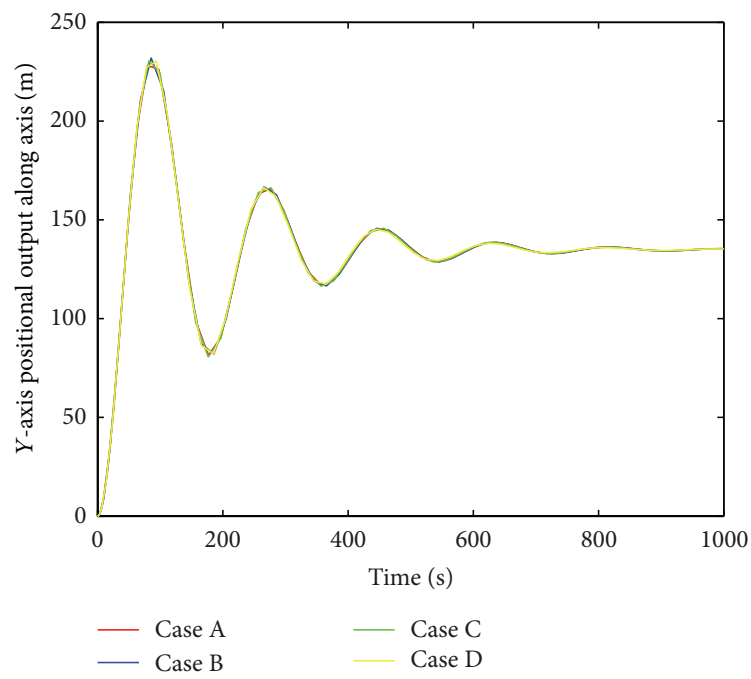

Figure 16: $Y$-axis positional output of closed-loop system for the four different uncertainty cases.

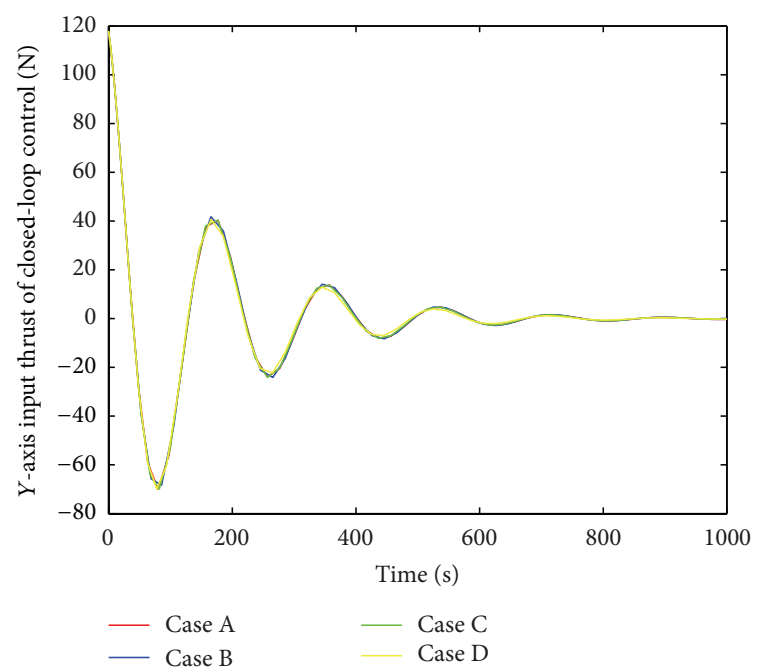

FIGURE 17: $Y$-axis input of closed-loop system for the four different uncertainty cases.

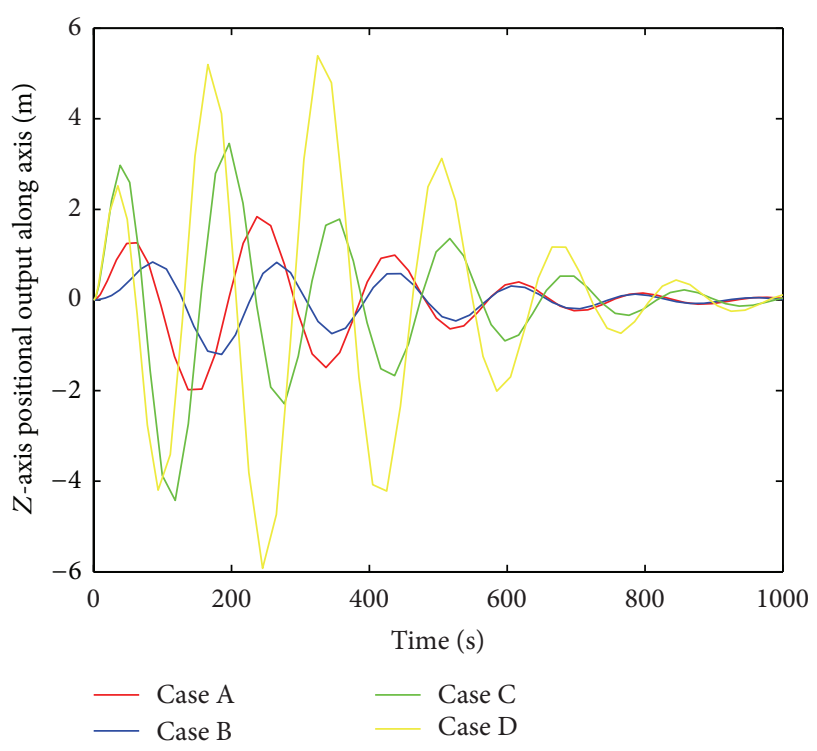

FIGURE 18: $Z$-axis positional output of closed-loop system for the four different uncertainty cases.

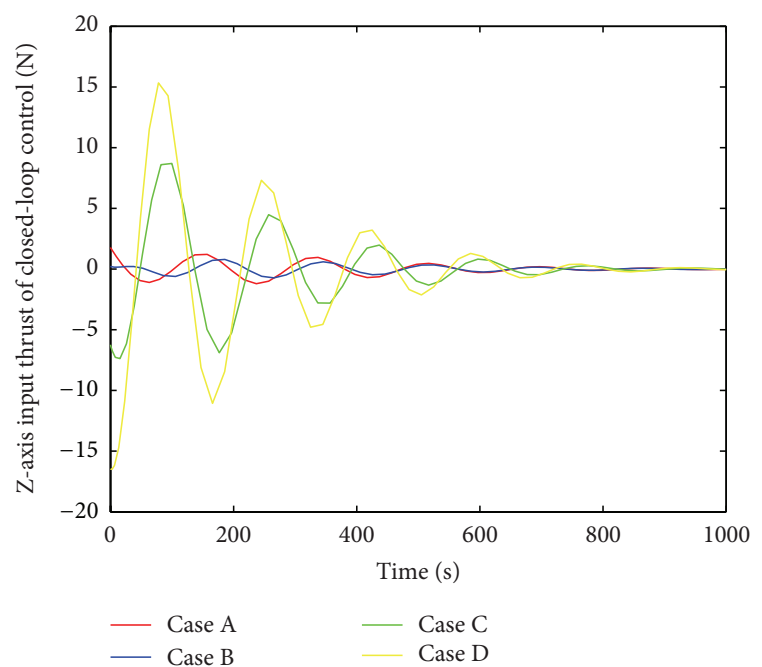

FIGURE 19: $Z$-axis input of closed-loop system for the four different uncertainty cases.

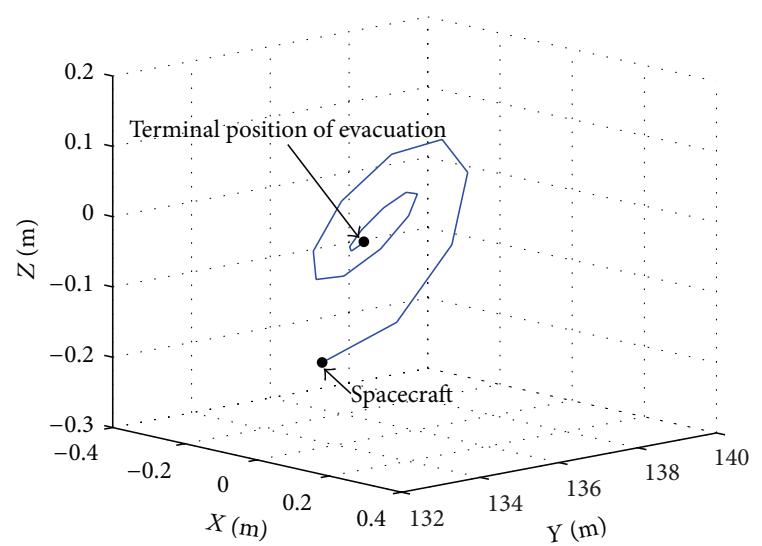

Figure 20: Terminal segment of the transfer orbit in rendezvous homing phase. 


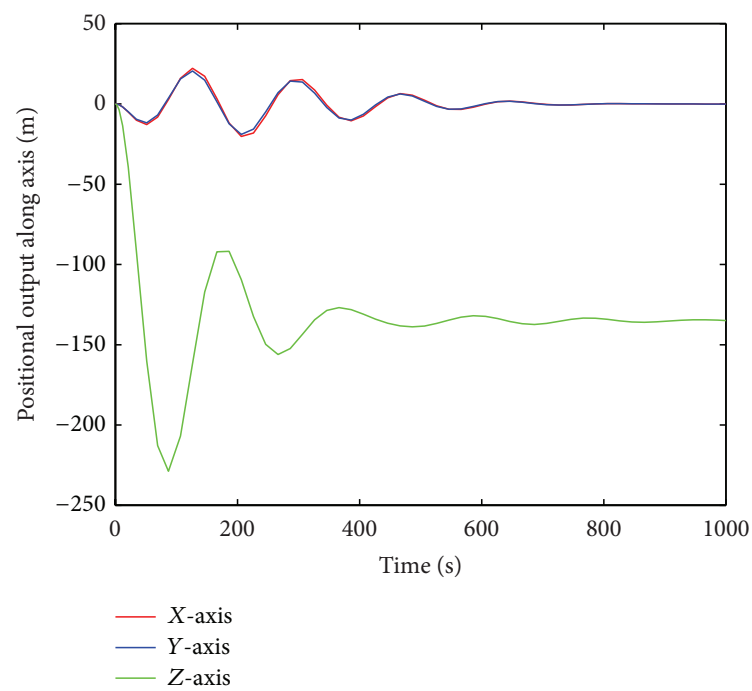

FIGURE 21: Chaser's positional output of closed-loop control.

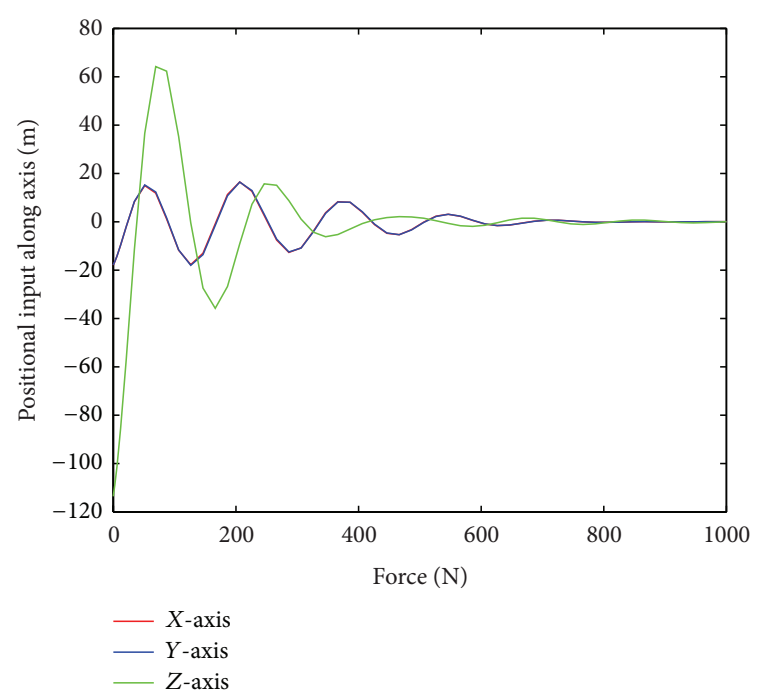

FIGURE 22: Chaser's input thrust of closed-loop control.

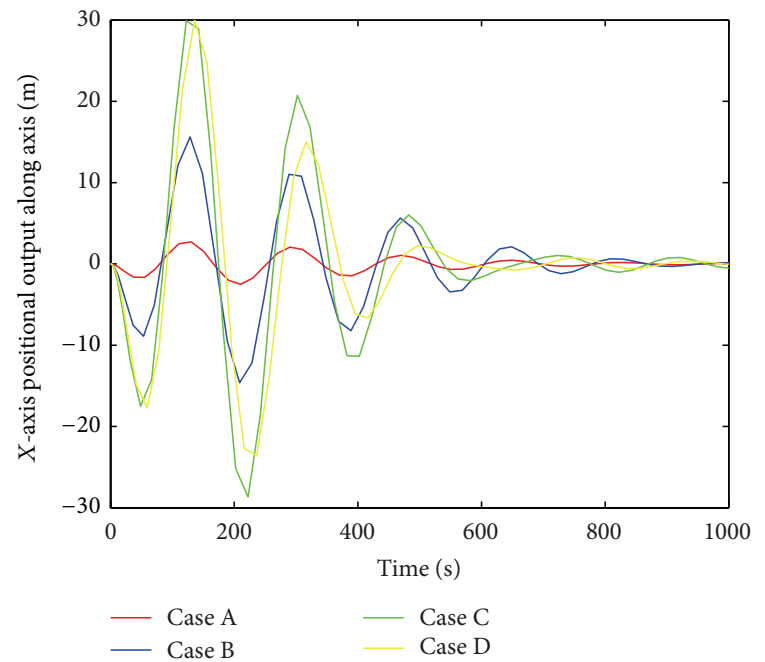

FIgUre 23: $X$-axis positional output of closed-loop system for the four different uncertainty cases.

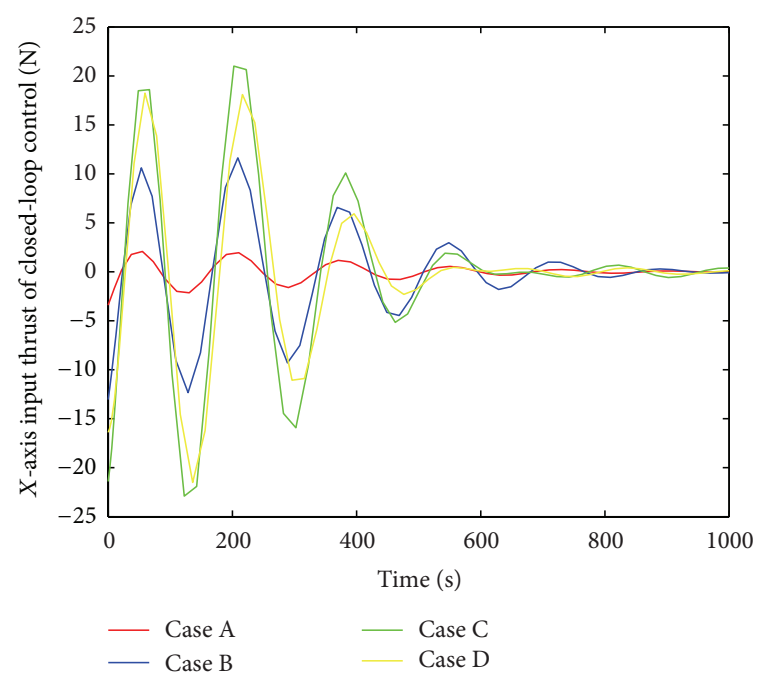

FIGURE 24: $X$-axis input of closed-loop system for the four different uncertainty cases.

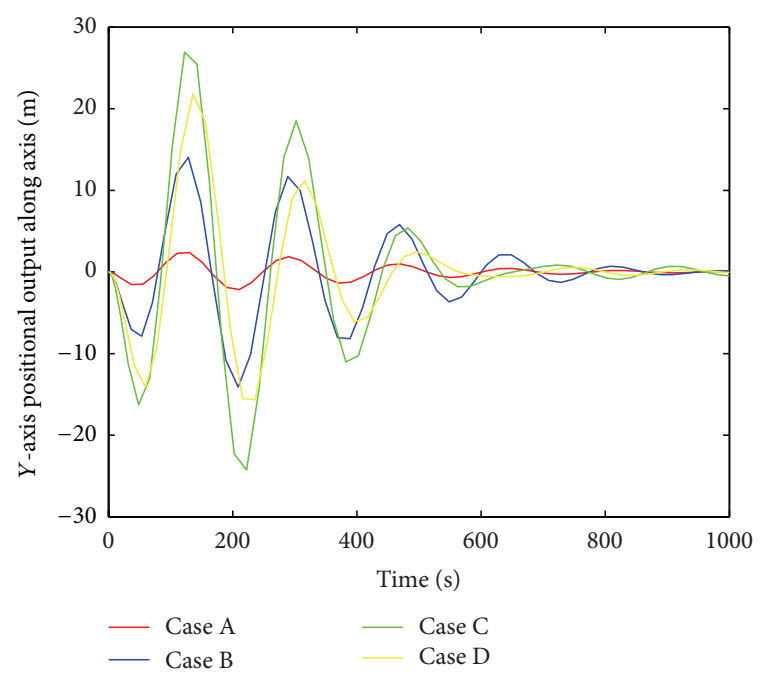

FIGURE 25: $Y$-axis positional output of closed-loop system for the four different uncertainty cases.

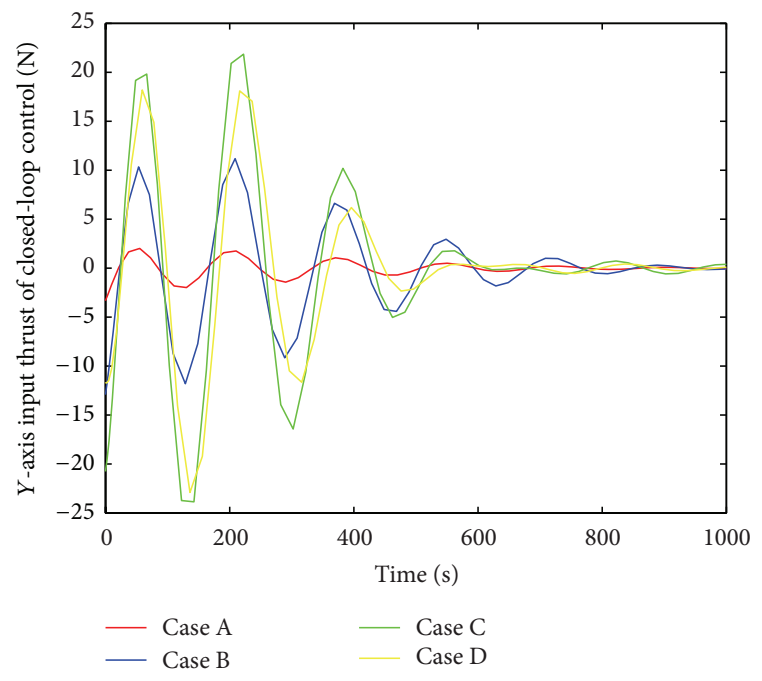

FIGURE 26: $Y$-axis input of closed-loop system for the four different uncertainty cases. 


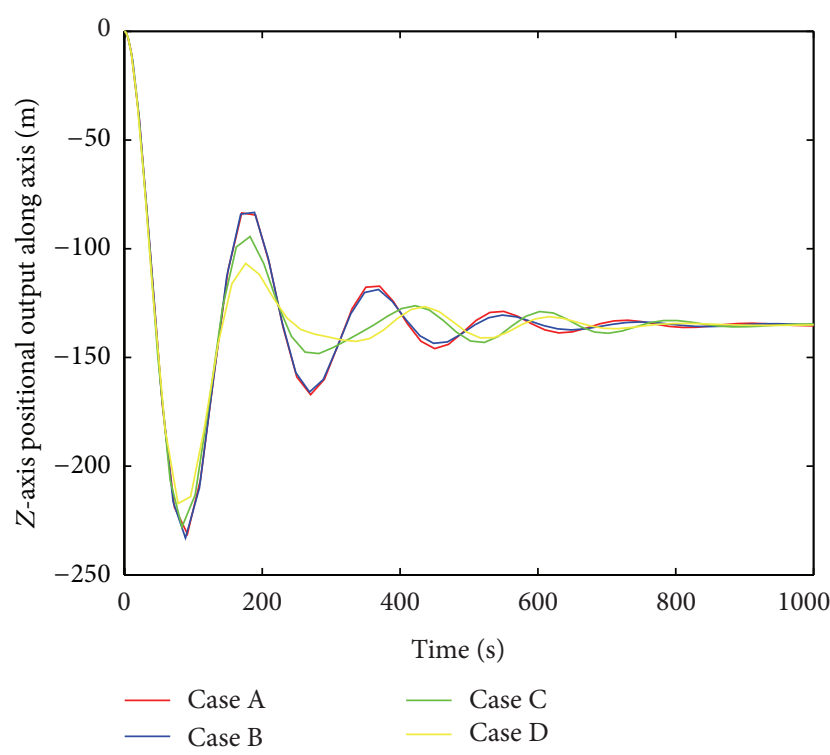

Figure 27: $Z$-axis positional output of closed-loop system for the four different uncertainty cases.

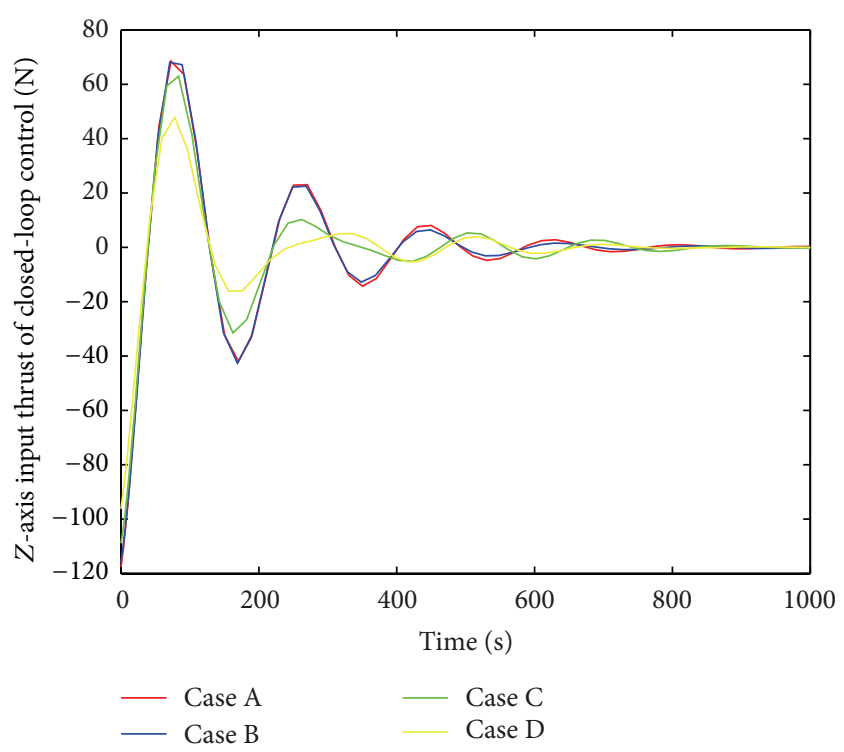

FIGURE 28: $Z$-axis input of closed-loop system for the four different uncertainty cases.

$$
\begin{aligned}
& K_{\text {Case A }}=\left[\begin{array}{ccccccccc}
-0.8726 & 0.0168 & -0.0012 & -19.6631 & -0.0165 & -0.0238 & -0.0156 & 0.0001 & -0.0001 \\
-0.0510 & -0.8183 & -0.1570 & -0.7472 & -22.4896 & -0.5307 & -0.0021 & -0.0140 & -0.0052 \\
-0.0232 & -0.1522 & -0.8247 & -1.0570 & -0.5274 & -22.3842 & -0.0012 & -0.0052 & -0.0144
\end{array}\right] \text {, } \\
& K_{\text {Case B }}=\left[\begin{array}{ccccccccc}
-0.8728 & 0.0137 & -0.0035 & -19.6836 & -0.0418 & -0.0430 & -0.0157 & -0.0001 & -0.0003 \\
-0.0886 & -0.7852 & -0.2359 & -1.5512 & -21.3489 & -1.2784 & -0.0029 & -0.0140 & -0.0074 \\
-0.0103 & -0.2165 & -0.7832 & -1.1239 & -0.8532 & -20.8999 & -0.0015 & -0.0071 & -0.0143
\end{array}\right] \text {, } \\
& K_{\text {Case C }}=\left[\begin{array}{ccccccccc}
-0.8723 & 0.0153 & -0.0014 & -19.7047 & -0.0793 & -0.0305 & -0.0156 & -0.0001 & -0.0002 \\
-0.1809 & -0.7652 & -0.2506 & -2.9144 & -20.5827 & -1.3472 & -0.0042 & -0.0138 & -0.0083 \\
0.0635 & -0.2401 & -0.7132 & -0.0714 & -0.5607 & -19.1985 & -0.0007 & -0.0075 & -0.0132
\end{array}\right] \text {, } \\
& K_{\text {Case D }}=\left[\begin{array}{ccccccccc}
-0.8702 & 0.0296 & 0.0013 & -19.6788 & -0.0089 & 0.0016 & -0.0154 & 0.0004 & 0.0000 \\
-0.3263 & -0.7475 & -0.1084 & -2.9313 & -22.6185 & -0.2819 & -0.0036 & -0.0106 & -0.0041 \\
0.2335 & 0.0512 & -0.7417 & 3.9454 & 4.0154 & -19.6471 & 0.0032 & -0.0009 & -0.0129
\end{array}\right] \text {, }
\end{aligned}
$$

and the positional outputs of the closed-loop systems with these controllers in three axes are depicted in Figures 23, 25 , and 27 separately. We can also obtain the four relevant control thrusts needed for these two methods in Figures 24, 26 , and 28 separately. We can see that the closed-loop systems with the controllers $K$ are all stable and the output tracking requirement can be guaranteed. From these figures and the table, we can also find that larger uncertainties will extend the stabilizing time and bring smaller fluctuations in $z$-axis input thrust, and the performance cost bound will also be diminished by larger uncertainties.

Finally, the terminal segment close to the target is depicted clearly in Figure 20. It can be seen that the chaser will asymptotically move to the evacuation point eventually, which means that the output tracking requirements can be satisfied by the designed controller $K$ (Figure 29).

\section{Conclusions}

The paper has demonstrated a robust guaranteed cost output tracking control design method for the evacuation phase of spacecraft rendezvous with parameter uncertainties. Tracking control problem has been altered into a stabilization problem of an augmented system by taking the reference signal of the output into consideration. By using Lyapunov method, the controller $K$ design problem has progressively been transformed into a convex optimization problem with linear matrix inequality constraints. The output tracking requirement can be satisfied with performance cost upper bound, and the poles of the augmented closed-loop system lie in the desired region. An illustrative example has shown the effectiveness of the proposed controller design method. 


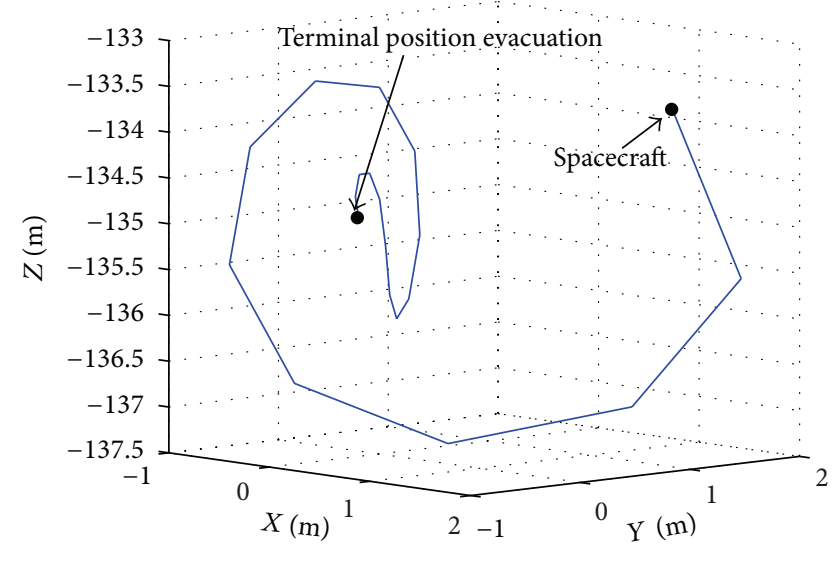

Figure 29: Terminal segment of the transfer orbit in rendezvous homing phase.

\section{Conflict of Interests}

The authors declare that there is no conflict of interests regarding the publication of this paper.

\section{Acknowledgment}

This work was partially supported by the National Natural Science Foundation of China (61203122), the China Postdoctoral Science Foundation (2012M510961), the Doctoral Fund of Ministry of Education of China (20122302120028), the Natural Science Foundation of Liaoning Province of China (2013020023) and the Key Laboratory Open Foundation of HIT (HIT.KLOF.2012.080).

\section{References}

[1] W. Fehse, Automated Rendezvous and Docking of Spacecraft, Cambridge University Press, Cambridge, UK, 2003.

[2] S. Yin, S. Ding, and H. Luo, "Real-time implementation of fault tolerant control system with performance optimization," IEEE Transactions on Industrial Electronics, vol. 61, no. 5, pp. 24022411, 2013.

[3] S. Yin, S. Ding, A. Haghani, H. Hao, and P. Zhang, "A comparison study of basic data-driven fault diagnosis and process monitoring methods on the benchmark Tennessee Eastman process," Journal of Process Control, vol. 22, no. 9, pp. 1567-1581, 2012.

[4] H. B. Hablani, M. L. Tapper, and D. J. Dana-Bashian, "Guidance algorithms for autonomous rendezvous of spacecraft with a target vehicle in circular orbit," Journal of Guidance, Control, and Dynamics, vol. 25, no. 3, pp. 553-562, 2002.

[5] G. Bergez, O. Mongrard, C. Santini, and R. Lainé, "ATV separation and departure strategy from uncontrolled international space station," in Proceedings of the 18th International Symposium on Space Flight Dynamics (ESA SP-548), pp. 85-90, Munich, Germany, October 2004.

[6] W. H. Clohessy and R. S. Wiltshire, "Terminal guidance system for satellite rendezvous," Journal of Aerospace Science, vol. 27, no. 9, pp. 653-658, 1960.
[7] W. H. Clohessy and R. S. Wiltshire, "Terminal guidance system for satellite rendezvous," in Proceedings of the American Institute of the Aerospace Sciences Summer Meeting, pp. 59-93, LosAngeles, Calif, USA, 1959.

[8] Y. Z. Luo and G. J. Tang, "Spacecraft optimal rendezvous controller design using simulated annealing," Aerospace Science and Technology, vol. 9, no. 8, pp. 732-737, 2005.

[9] D. J. Jezewski and J. D. Donaldson, "An analytical approach to optimal rendezvous using Clohessy-Wiltshire equations," Journal of the Astronautical Sciences, vol. 27, no. 3, pp. 293-310, 1979.

[10] Y. Z. Luo, G. J. Tang, and Y. J. Lei, “Optimal multi-objective linearized impulsive rendezvous," Journal of Guidance, Control, and Dynamics, vol. 30, no. 2, pp. 383-389, 2007.

[11] G. L. Tang, Y. Z. Luo, and H. Y. Li, “Optimal robust linearized impulsive rendezvous," Aerospace Science and Technology, vol. 11, no. 7-8, pp. 563-569, 2007.

[12] S. Yin, S. Ding, A. Haghani, and H. Hao, "Data-driven monitoring for stochastic systems and its application on batch process," International Journal of Systems Science, vol. 44, no. 7, pp. 13661376, 2013.

[13] M. Basin and D. Calderon-Alvarez, "Optimal LQG controller for linear stochastic systems with unknown parameters," Journal of the Franklin Institute, vol. 345, no. 3, pp. 293-302, 2008.

[14] M. Basin and D. Calderon-Alvarez, "Optimal filtering over linear observations with unknown parameters," Journal of the Franklin Institute, vol. 347, no. 6, pp. 988-1000, 2010.

[15] H. Gao and C. Wang, "A delay-dependent approach to robust $H_{\infty}$ filtering for uncertain discrete-time state-delayed systems," IEEE Transactions on Signal Processing, vol. 52, no. 6, pp. 16311640, 2004.

[16] P. Shi, E. Boukas, and R. K. Agarwal, "Control of Markovian jump discrete-time systems with norm bounded uncertainty and unknown delay," IEEE Transactions on Automatic Control, vol. 44, no. 11, pp. 2139-2144, 1999.

[17] J. Wang, P. Shi, H. Gao, and J. Wang, "Gain-scheduled stabilisation of linear parameter-varying systems with time-varying input delay," IET Control Theory and Applications, vol. 1, no. 5, pp. 1276-1285, 2007.

[18] J. Zhang, P. Shi, and J. Qiu, "Non-fragile guaranteed cost control for uncertain stochastic nonlinear time-delay systems," Journal of the Franklin Institute, vol. 346, no. 7, pp. 676-690, 2009.

[19] I. R. Petersen, "A stabilization algorithm for a class of uncertain linear systems," Systems and Control Letters, vol. 8, no. 4, pp. 351357, 1987.

[20] P. Singla, K. Subbarao, and J. L. Junkins, "Adaptive output feedback control for spacecraft rendezvous and docking under measurement uncertainty," Journal of Guidance, Control, and Dynamics, vol. 29, no. 4, pp. 892-902, 2006.

[21] B. Ebrahimi, M. Bahrami, and J. Roshanian, “Optimal slidingmode guidance with terminal velocity constraint for fixedinterval propulsive maneuvers," Acta Astronautica, vol. 62, no. 10-11, pp. 556-562, 2008.

[22] G. W. Hughes and C. R. McInnes, "Solar sail hybrid trajectory optimization for non-Keplerian orbit transfers," Journal of Guidance, Control, and Dynamics, vol. 25, no. 3, pp. 602-604, 2002.

[23] C. A. Kluever, "Optimal low-thrust interplanetary trajectories by direct method techniques," Journal of the Astronautical Sciences, vol. 45, no. 3, pp. 247-262, 1997. 
[24] C. A. Kluever, "Comet rendezvous mission design using solar electric propulsion spacecraft," Journal of Spacecraft and Rockets, vol. 37, no. 5, pp. 698-700, 2000.

[25] G. Mengali and A. A. Quarta, "Fuel-optimal, power-limited rendezvous with variable thruster efficiency," Journal of Guidance, Control, and Dynamics, vol. 28, no. 6, pp. 1194-1199, 2005.

[26] D. S. Naidu, "Fuel-optimal trajectories of aeroassisted orbital transfer with plane change," IEEE Transactions on Aerospace and Electronic Systems, vol. 27, no. 2, pp. 361-369, 1991.

[27] W. A. Scheel and B. A. Conway, "Optimization of verylow-thrust, many-revolution spacecraft trajectories," Journal of Guidance, Control, and Dynamics, vol. 17, no. 6, pp. 1185-1192, 1994.

[28] X. Yang, H. Gao, and P. Shi, "Robust orbital transfer for low earth orbit spacecraft with small-thrust," Journal of the Franklin Institute, vol. 347, no. 10, pp. 1863-1887, 2010.

[29] X. Yang, X. Cao, and H. Gao, "Sampled-data control for relative position holding of spacecraft rendezvous with thrust nonlinearity," IEEE Transactions on Industrial Electronics, vol. 59, no. 2, pp. 1146-1153, 2012.

[30] X. Yang and H. Gao, "Guaranteed cost output tracking control for autonomous homing phase of spacecraft rendezvous," Journal of Aerospace Engineering, vol. 24, no. 4, pp. 478-487, 2011.

[31] L. Yu and J. Chu, "LMI approach to guaranteed cost control of linear uncertain time-delay systems," Automatica, vol. 35, no. 6, pp. 1155-1159, 1999. 


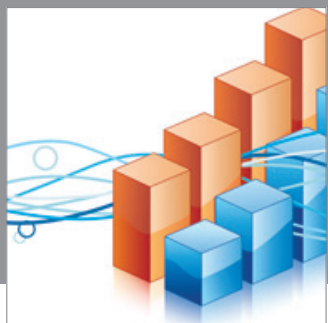

Advances in

Operations Research

mansans

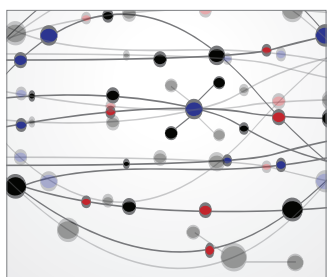

The Scientific World Journal
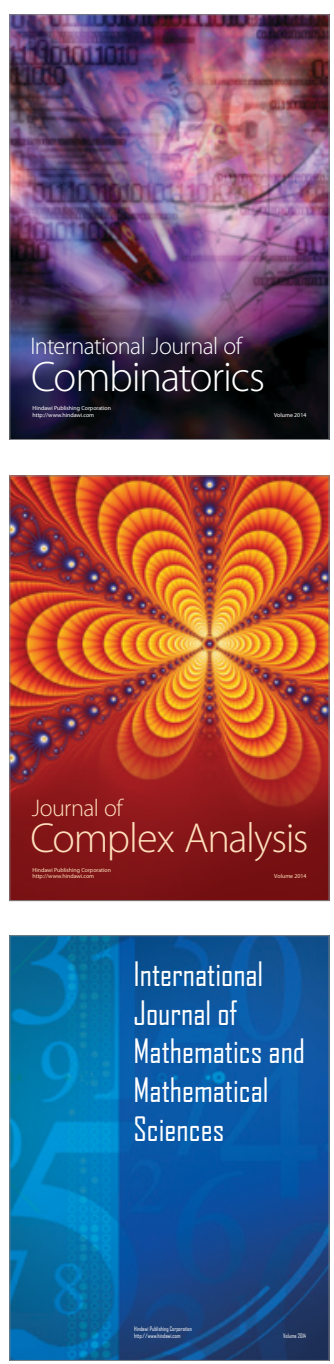
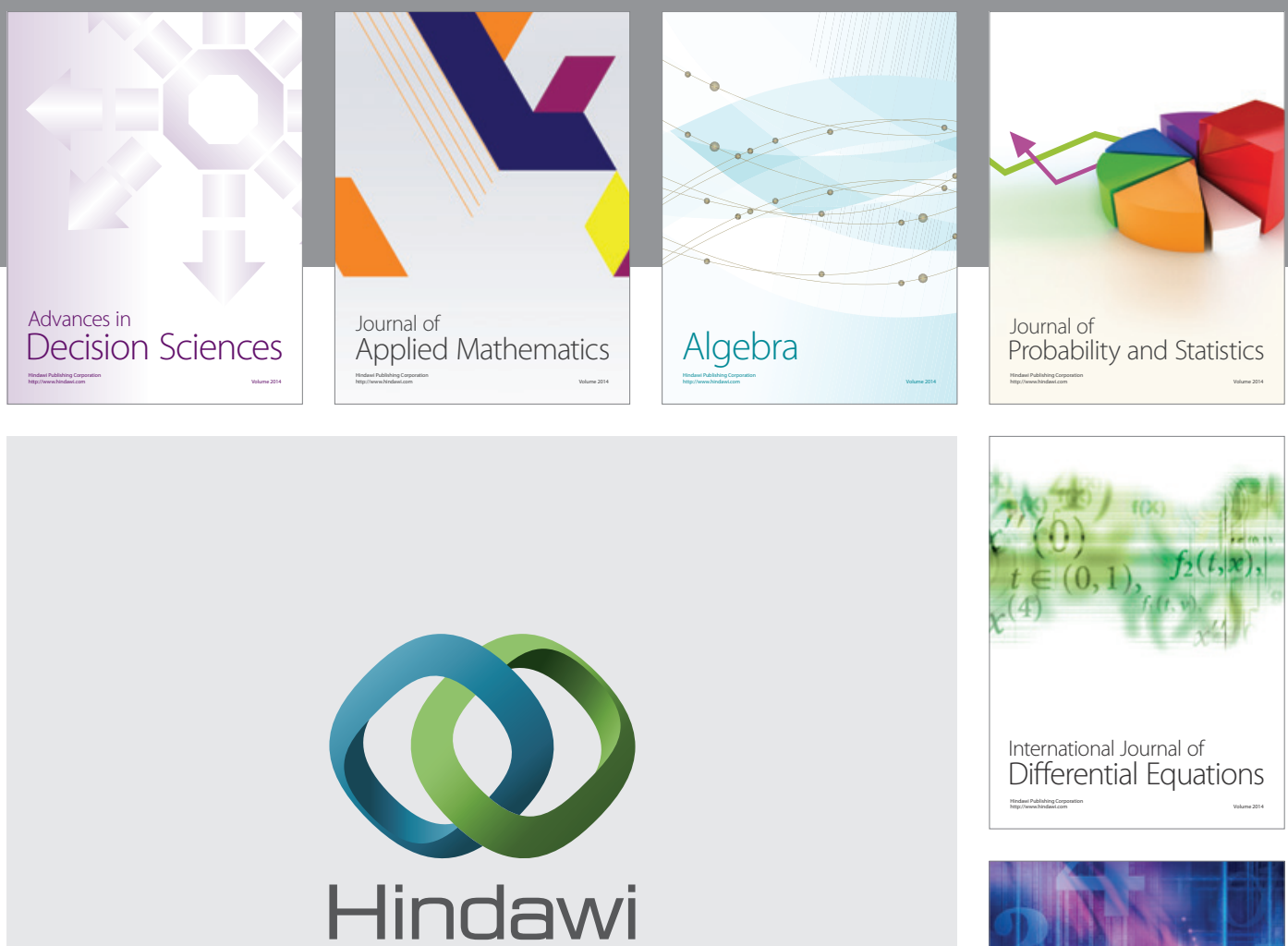

Submit your manuscripts at http://www.hindawi.com
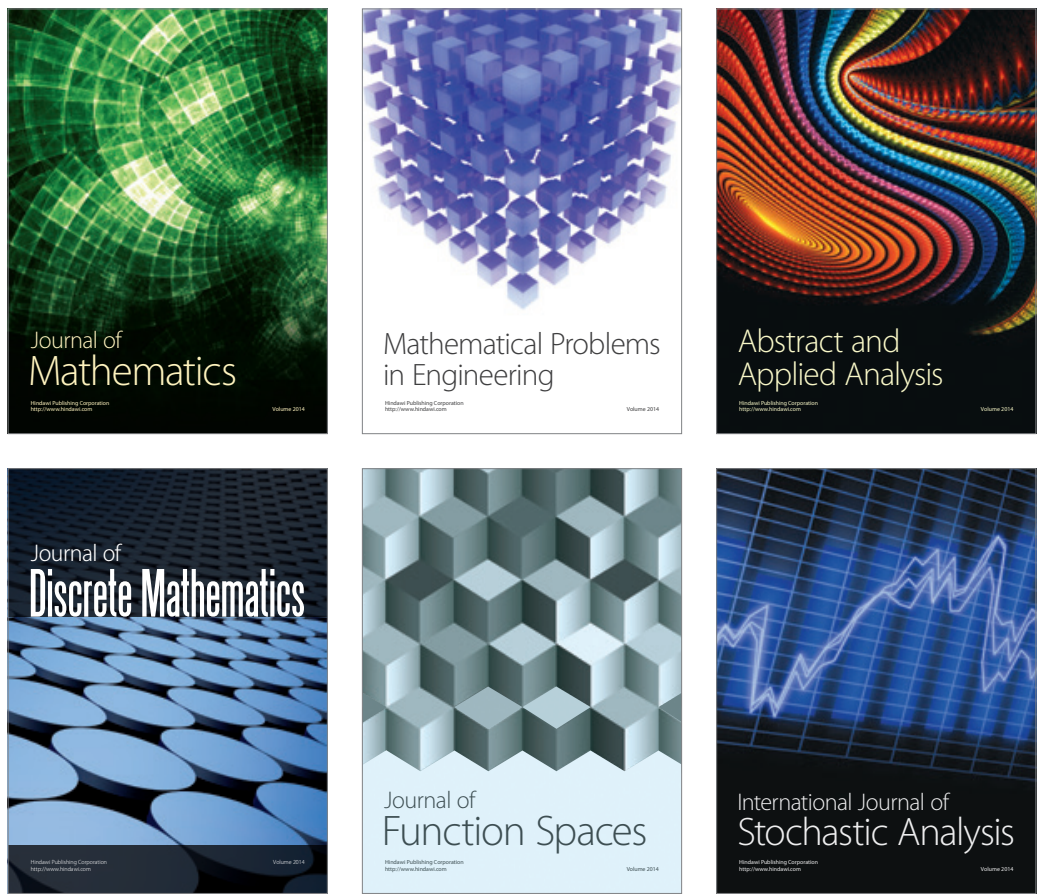

Journal of

Function Spaces

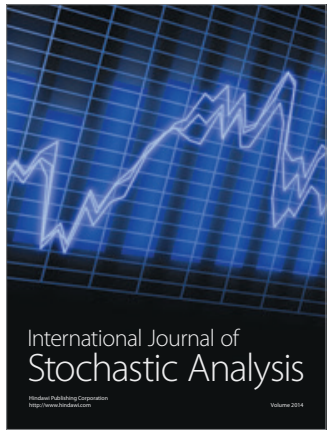

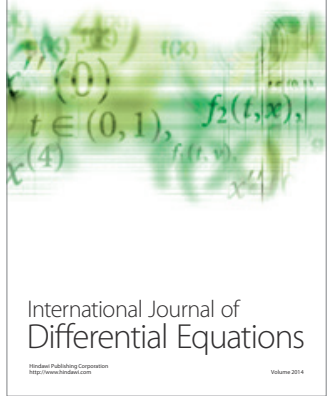
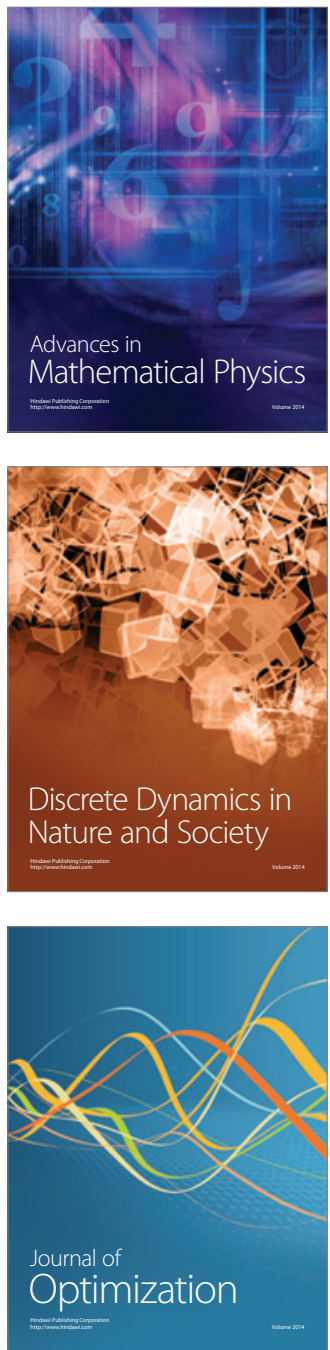